\title{
Aspects of renormalization in finite-density field theory
}

\author{
A. Liam Fitzpatrick, ${ }^{1,2}$ Gonzalo Torroba, ${ }^{3}$ and Huajia Wang ${ }^{1}$ \\ ${ }^{1}$ Stanford Institute for Theoretical Physics, Stanford University, Stanford, California 94305, USA \\ ${ }^{2}$ SLAC National Accelerator Laboratory, 2575 Sand Hill Road, Menlo Park, California 94025, USA \\ ${ }^{3}$ Centro Atómico Bariloche and CONICET, R8402AGP Bariloche, Argentina
}

(Received 4 March 2015; revised manuscript received 8 May 2015; published 26 May 2015)

\begin{abstract}
We study the renormalization of the Fermi surface coupled to a massless boson near three spatial dimensions. For this, we set up a Wilsonian RG with independent decimation procedures for bosons and fermions, where the four-fermion interaction "Landau parameters" run already at tree level. Our explicit one-loop analysis resolves previously found obstacles in the renormalization of finite-density field theory, including logarithmic divergences in nonlocal interactions and the appearance of multilogarithms. The key aspects of the RG are the above tree-level running, and a UV-IR mixing between virtual bosons and fermions at the quantum level, which is responsible for the renormalization of the Fermi velocity. We apply this approach to the renormalization of $2 k_{F}$ singularities, and to Fermi surface instabilities in a companion paper, showing how multilogarithms are properly renormalized. We end with some comments on the renormalization of finite-density field theory with the inclusion of Landau damping of the boson.
\end{abstract}

\section{INTRODUCTION}

Quantum field theory (QFT) at finite density appears as the continuum limit in a wide array of systems, ranging from neutron stars to novel quantum critical points in condensed matter physics. Its quantum properties are, however, much less well understood than in the relativistic case. In particular, additional subtleties arise when the Fermi surface is coupled to gapless modes. In the present work we focus on the renormalization of such theories, with the goal of setting up a consistent renormalization group (RG) approach.

Some of the most interesting applications of finite density occur at strong coupling, e.g., as models of strongly correlated electron systems; however, it has been hard to identify a general framework where finite-density field theories at strong coupling can be understood. On the other hand-and we will see this explicitly below-weakly coupled limits already exhibit very interesting physics and challenge standard RG ideas. From experience in relativistic theories, understanding in detail the perturbative limits is a key to the strong-coupling problem. Indeed, one can try to identify properties of finitedensity field theory at weak coupling that extend to the more general case, for instance by use of an $\epsilon$ expansion or in certain large- $N$ limits.

In this work we will study the renormalization of finitedensity field theory at weak coupling and in a small $\epsilon$ expansion, continuing the analysis started in [1-3]. The class of theories that we will focus on contains massless bosons (scalars or gauge fields) coupled to fermions at finite density by a Yukawa interaction of the form $g \phi \psi^{\dagger} \psi$. We will work near $d=3$ spatial dimensions, the upper critical dimensional where the coupling $g$ is marginal and a weak-coupling expansion can be set up.

The coupled system of a critical boson interacting with a Fermi surface is not fully understood even at weak coupling, and requires going beyond the Fermi liquid RG setup in $[4,5]$. The main new effect of finite density is that bosons and fermions have very different energetics and scalings. Low-energy bosonic excitations occur near $\vec{k}=0$; therefore, boson momenta scale towards the origin at low energies. In contrast, IR fermionic excitations occur at the Fermi surface of finite momenta $\vec{k}=\vec{k}_{F}$. When these two sets of degrees of freedom are allowed to interact, novel quantum effects arise, which are absent in the relativistic theory or in models with only fermions at finite density.

Our task is to determine a consistent RG for the coupled system of bosons and finite-density fermions. Important first steps were taken in developing such an RG in the seminal works on color superconductivity in QCD at finite density $[6,7] .{ }^{1}$ A key result in that approach, which we make use of as well, is the existence of logarithmic divergences in 4-Fermi interactions already at tree level. However, going beyond the leading order analysis in [6,7], many new subtleties arise. The main goal of this paper is to develop a fully systemic Wilsonian approach that may be used to higher orders and that addresses the nontrivial issues that arise there. In particular, our proposal will resolve two problems that made the renormalization of systems of coupled bosons and finite-density fermions quite involved: the nonlocal (singular) logarithmic divergences recently found in [3], and the presence of multilogarithms in 4-Fermi interactions from exchange of massless bosons. We will show the tree-level running of 4Fermi interactions explicitly in the calculation of the Wilsonian action, together with the required tree-level counterterms. In terms of these running couplings we will argue that the theory is renormalizable. We will then apply this $\mathrm{RG}$ to calculate quantum corrections to Landau parameters and the $2 k_{F}$ vertex, showing how previous problems in their renormalization are resolved. An RG analysis of Fermi surface instabilities will be presented in the companion paper [9].

First, in Sec. II D we present the RG approach, explaining the decimation procedures, the origin of UV-IR mixing in the presence of massless bosons, and the tree-level running of the

\footnotetext{
${ }^{1}$ This approach has recently been applied to theories of bosons coupled to the Fermi surface in [8].
} 
Landau parameters. Next, Sec. III contains our main results at one loop. We prove the cancellation of divergences in all (marginally) relevant interactions and compute the RG $\beta$ functions. These results are then applied in Sec. IV to the study of the RG flow for the Landau parameters and for the $2 k_{F}$ vertex. Lastly, Sec. V briefly discusses the possible extension to higher loop order, with the inclusion of Landau damping of the boson. We end by discussing interesting future directions in Sec. VI. In the Appendix we perform a detailed analysis of the theory in dimensional regularization, a regulator that is efficient in correctly capturing the various quantum effects and that will also be needed in future higher loop extensions of our work.

\section{SCALING AND RENORMALIZATION AT TREE LEVEL}

In this first section we will discuss some general properties of renormalization of quantum field theories where bosons interact with a finite density of fermions. Applying the renormalization group to finite-density theories is quite challenging for several reasons. First, the scaling properties of bosons and fermions are now very different: low-energy bosonic excitations are located near the origin of momentum space, while for fermions they occur around the Fermi surface $\vec{k}=\vec{k}_{F}$. Second, the Fermi surface leads to enhanced quantum contributions from the large number of light degrees of freedom. This enhancement can compensate for the phase suppression factor from momentum integration, with the result that low-energy excitations will also contribute to the $\mathrm{RG}$ evolution of the theory. The Wilsonian RG of Shankar and Polchinski [4,5], where fermion shells of high momentum are integrated out, needs to be modified. Our task is to set up an $\mathrm{RG}$ that can deal consistently with these problems.

\section{A. Classical theory}

For concreteness we will consider a real spin zero boson interacting with a spinless fermion,

$$
\begin{aligned}
S= & \int d \tau d^{d} x\left\{\frac{1}{2}\left[\left(\partial_{\tau} \phi\right)^{2}+c^{2}(\vec{\nabla} \phi)^{2}\right]\right. \\
& \left.+\psi^{\dagger}\left[\partial_{\tau}+\varepsilon_{F}(i \vec{\nabla})-\mu_{F}\right] \psi+L_{\text {int }}\right\},
\end{aligned}
$$

where $\varepsilon_{F}(\vec{k})$ is the quasiparticle energy and $\mu_{F}$ is the chemical potential. The basic property of the fermion energy function $\varepsilon(\vec{k}) \equiv \varepsilon_{F}(\vec{k})-\mu_{F}$ is that it admits a Fermi surface at finite momentum, $\varepsilon\left(\vec{k}_{F}\right)=0$. For instance, $\varepsilon(\vec{k})=\vec{k}^{2} / 2 m-\mu_{F}$ for a massive fermion at finite chemical potential $\mu_{F}$. Our analysis can be easily extended to other fields, such as gauge bosons and Dirac fermions. Given this matter content, possible interaction terms include a boson-fermion Yukawa coupling, as well as 4-Fermi and $\phi^{4}$ couplings,

$$
L_{\text {int }}=g \phi \psi^{\dagger} \psi+\lambda\left(\psi^{\dagger} \psi\right)^{2}+\lambda^{\prime} \phi^{4}+\cdots .
$$

These interactions can depend on momenta, and we will define them in more detail shortly. The classical $\phi^{4}$ coupling will not play an important role, so we set $\lambda^{\prime}=0$. We also set the boson speed $c=1$.

In order to study the low-energy theory near the Fermi surface in its simplest form, it will be convenient to assume a

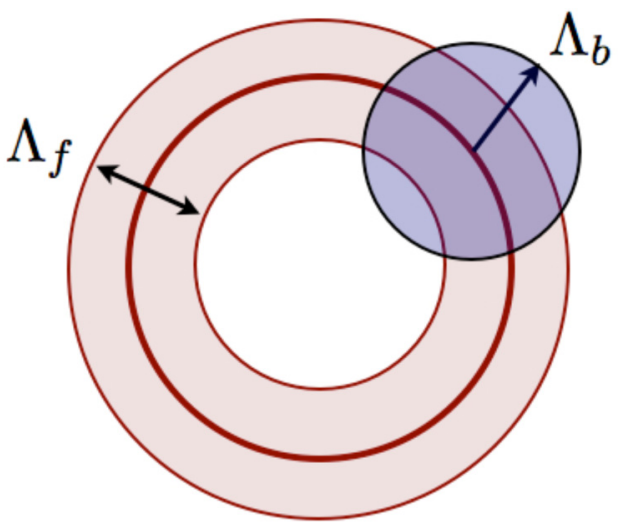

FIG. 1. (Color online) Scaling towards low energies for bosons (in terms of $\Lambda_{b}$ ) and fermions (for $\Lambda_{f}$ ). Low-energy bosonic modes occur near the origin, while for fermions they arise around the Fermi surface.

spherical Fermi surface. Following the spherical RG of [4,5], the fermion momentum is measured radially from the Fermi surface,

$$
\vec{k}=\hat{n}\left(k_{F}+k_{\perp}\right)
$$

with $\hat{n}$ a unit vector perpendicular to the Fermi surface. In the low-energy theory $\omega \ll \mu_{F}, k_{\perp} \ll k_{F}$, it is sufficient to expand

$$
\begin{aligned}
S_{f}= & \int d \tau \frac{d \Omega_{n}}{(2 \pi)^{d-1}} \frac{d k_{\perp}}{2 \pi} \psi^{\dagger}(\vec{k}) \\
& \times\left(\partial_{\tau}+v k_{\perp}+\frac{w}{2 k_{F}} k_{\perp}^{2}+\cdots\right) \psi(\vec{k}) .
\end{aligned}
$$

Here we have rescaled the fermion to absorb an overall power of $k_{F}$.

We will see that in the effective theory at energies much smaller than the Fermi energy $\mu_{F}$, quadratic and higher order corrections to the dispersion relation can be neglected. It will then be sufficient to keep the leading linear term, working with a flat band of Fermi velocity $v=\varepsilon_{F}^{\prime}\left(\vec{k}_{F}\right)$. In this case, our results will also apply locally on the Fermi surface, even if the surface is not spherical (as long as it is smooth). This local approximation, which will be useful below, should not be confused with the patch RG (see, e.g., [10,11]), where normal and tangential directions in the patch have different scalings.

\section{B. Renormalization approach}

Because of the very different boson and fermion scalings, it is natural to define an effective theory that depends on two independent cutoffs $\Lambda_{b}$ and $\Lambda_{f}$. One cutoff $\Lambda_{b}$ regulates the scaling of the bosonic degrees of freedom towards the origin, $p^{2}<\Lambda_{b}$; the other cutoff $|\varepsilon(p)|<\Lambda_{f}$ dictates the displacement from the Fermi surface in the low-energy theory. The two scales can be varied independently, and as a result it is possible to have different decimation procedures for bosons and fermions. This is illustrated in Fig. 1 for a spherical Fermi surface. 
A smooth version of these cutoffs is obtained by modifying the propagators of bosons and fermions in terms of a function $K(x)$,

$$
D(p)=\frac{K\left(\vec{p}^{2} / \Lambda_{b}^{2}\right)}{p_{0}^{2}+\vec{p}^{2}}, \quad G(p)=-\frac{K\left(\varepsilon(\vec{p})^{2} / \Lambda_{f}^{2}\right)}{i p_{0}-\varepsilon(\vec{p})},
$$

with $K(x) \rightarrow 1$ for $x \ll 1$ and $K(x) \rightarrow 0$ for $x \gg 1$; the boson speed has been set to $c=1$. We furthermore choose regulators that do not constrain the frequency, which will be integrated over the whole range $(-\infty, \infty){ }^{2}$ Under an infinitesimal variation of the cutoffs $\delta \Lambda_{b, f}$, the action changes in order to keep the partition function fixed $[12,13]$, defining a bidimensional RG flow. Having these two cutoffs will also allow us to distinguish between local and nonlocal renormalization effects, as well as the origin of multilogarithms [9]. A tree-level RG for the coupled boson-fermion system similar to that suggested in Fig. 1 was studied in [14]. It is interesting to note that two-dimensional RG flows also appear for jets in QCD; see, e.g., $[15,16]$.

In the effective theory with the two cutoffs $\Lambda_{b}$ and $\Lambda_{f}$, fermions can scatter with a maximum exchanged momentum set by $\Lambda_{b}$. As a result, it will be sufficient to restrict to a local patch (or two antipodal patches) of the Fermi surface of angular size $\Lambda_{b} / k_{F}$, as shown in Fig. 1. We will return to this point below in Sec. II D. A basic approximation that we will use throughout this work is that we will linearize the fermion dispersion relation, effectively neglecting quadratic and higher order terms in $\varepsilon(\vec{p})$. This will be valid over the whole patch as long as

$$
\Lambda_{b} \lesssim \sqrt{2 k_{F} \Lambda_{f}},
$$

a relation that we will assume in what follows. ${ }^{3}$

A problem with momentum cutoffs is that they can break Ward identities and hence may require additional counterterms. In our case a finite-density analog of the Ward identity of $U(1)$ charge will play a useful guiding role, and we wish to preserve it. For this reason, in the renormalization of the theory it can be convenient to use a dimensional regularization (DR) procedure that preserves gauge invariance, taking the limit $\epsilon \rightarrow 0$ in $d=3-\epsilon$. However, due to its conceptual transparency, we will mostly use the hard cutoffs in the body of the paper, and describe renormalization using DR in Appendix A.

Computationally, the theory will be renormalized following the standard QFT procedure of writing the bare fields and couplings (denoted by a subindex " 0 ") in terms of physical quantities plus counterterms. For the action (2.1), the required

\footnotetext{
${ }^{2}$ One could alternately regulate the frequency range as well. Some Feynman integrals in the theory are finite and yet still dependent on the ratio of the frequency cutoff to momentum cutoff, and so different choices correspond to different choices for local counterterms. We caution the reader that this can lead to additional scheme-dependence of $\beta$ functions at higher loops beyond what one might perhaps be used to.

${ }^{3} \mathrm{We}$ are assuming that $v$ and $w$ are comparable in (2.4), as is the case for an approximately quadratic dispersion relation. Otherwise an extra factor of $v / w$ is required in (2.6).
}

redefinitions are

$$
\begin{aligned}
& \psi_{0}=Z_{\psi}^{1 / 2} \psi, \quad \phi_{0}=Z_{\phi}^{1 / 2} \phi, \quad v_{0}=Z_{v} v, \\
& g_{0}=\mu^{\epsilon / 2} \frac{Z_{g}}{Z_{\phi}^{1 / 2} Z_{\psi}} g, \quad \lambda_{0}=\frac{Z_{\lambda}}{Z_{\psi}^{2}} \lambda
\end{aligned}
$$

where $\mu$ is an arbitrary RG scale and $g$ and $\lambda$ are dimensionless. The factors $Z_{i}$ are adjusted to cancel divergences, and the $\beta$ functions are calculated noting that the bare parameters are $\mu$ independent. Below we will work in terms of counterterms $\delta_{i}$ defined as

$$
\begin{aligned}
Z_{\psi} & =1+\delta_{\psi}, \quad Z_{v} v=v+\delta v, \\
Z_{g} g & =g+\delta g, \quad Z_{\lambda} \lambda=\lambda+\delta \lambda .
\end{aligned}
$$

\section{UV-IR mixing}

Let us explain, at this general level, how the Fermi surface leads to enhanced quantum contributions from low-energy excitations. These effects will play an important role in the renormalization of the velocity and the boson-fermion cubic coupling. For this, we note that close to the Fermi surface, the product of two fermion propagators with momenta $p$ and $p+q$ has the structure

$$
G(p) G(p+q) \approx G(p)^{2}+2 \pi \frac{\operatorname{sgn}(v) q_{\perp}}{i q_{0}-v q_{\perp}} \delta\left(p_{0}\right) \delta\left(p_{\perp}\right),
$$

in the limit $q \rightarrow 0$ and in the cutoff regulator that we just introduced. ${ }^{4}$ This can be seen by integrating both sides over $p_{0}$ and $p_{\perp}$. More explicitly, integrating $p_{0}$ by residues in the left-hand side obtains

$$
\begin{aligned}
& \int \frac{d p_{0} d p_{\perp}}{\left(i p_{0}-v p_{\perp}\right)\left[i\left(p_{0}+q_{0}\right)-v\left(p_{\perp}+q_{\perp}\right)\right]} \\
& \quad=\frac{2 \pi \operatorname{sgn}(v)}{i q_{0}-v q_{\perp}} \int d p_{\perp}\left[\Theta\left(p_{\perp}+q_{\perp}\right)-\Theta\left(p_{\perp}\right)\right] \\
& \quad=2 \pi \frac{\operatorname{sgn}(v) q_{\perp}}{i q_{0}-v q_{\perp}},
\end{aligned}
$$

and this reproduces the integral of the right-hand side in (2.9). The consequences of this for the scattering of quasiparticles and properties of collective excitations are well known; see, e.g., the discussion in [17].

In Fermi liquids without gapless bosons, the singular second term in (2.9) does not contribute to the RG and is not included. However, when the system is coupled to a gapless boson, such singular terms in the product of fermion lines can lead to logarithmic enhancements from the exchange of virtual boson. An important example is the one-loop correction to the cubic vertex, proportional to

$$
\int_{p} D(p) G(p) G(p+q)
$$

\footnotetext{
${ }^{4}$ We note that the integral $\int_{p} G(p) G(p+q)$ is ambiguous in the low-energy theory and it depends on the ratio of the frequency and momentum cutoffs. Here we are taking the frequency cutoff to infinity first.
} 


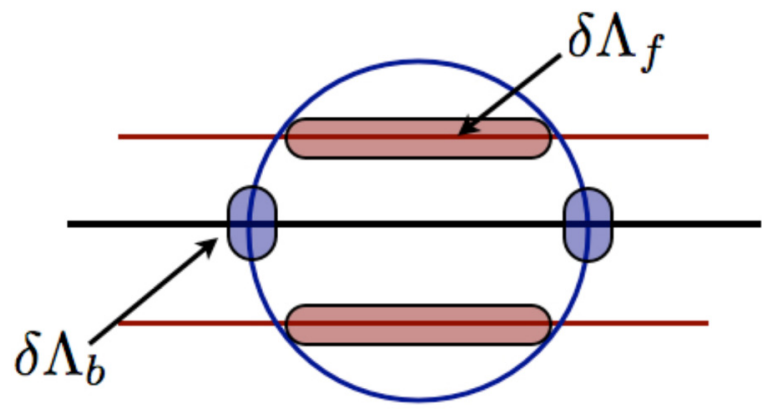

FIG. 2. (Color online) Dominant contributions to the RG for the Fermi surface coupled to a gapless boson. A local patch of the Fermi surface (here shown in black) is delimited by $\Lambda_{b}$, while the width of low-energy excitations around the surface is controlled by $\Lambda_{f}$. By varying these cutoffs, the dominant quantum contributions come from the two shells in the figure determined by $\delta \Lambda_{f}$ and $\delta \Lambda_{b}$.

The second term in (2.9), when multiplying the boson propagator, produces an extra logarithmic divergence,

$$
\int_{p} D(p) \frac{q_{\perp}}{i q_{0}-v q_{\perp}} \delta\left(p_{0}\right) \delta\left(p_{\perp}\right) \sim \frac{q_{\perp}}{i q_{0}-v q_{\perp}} \int \frac{d^{2} p_{\|}}{p_{\|}^{2}},
$$

which has to be taken into account in the renormalization of the theory. ${ }^{5}$ This is a UV-IR mixing, where low-energy fermionic excitations can exchange high-energy bosonic modes, contributing, as we shall see, to the running of couplings in the effective theory.

As a result, we will find that quantum effects are dominated by two regions of momenta near the Fermi surface, as shown in Fig. 2. One class of logarithms will come from the standard RG towards the Fermi surface and will be regulated by $\Lambda_{f}$. The other contributions will be proportional to $\ln \Lambda_{b}$, and will be generated by virtual bosons as in (2.12); these effects appear due to the $\delta$ function peak in the product of two fermion lines at the Fermi surface.

\section{Tree-level RG for 4-Fermi interactions}

The first contribution to the RG occurs already at tree level, in the form of a logarithmic running of 4-Fermi interactions due to the exchange of virtual bosons [6-8]. We will now discuss in detail how this comes about, which will be the basis for renormalization at the loop level.

Let us first recall some properties of 4-Fermi interactions, which we write as

$$
\begin{aligned}
S_{4-\text { Fermi }}= & -\int \prod_{i} d^{d+1} k_{i} \lambda\left(k_{4}, \ldots, k_{1}\right) \delta^{d+1}\left(k_{1}+k_{2}-k_{3}-k_{4}\right) \\
& \times \psi\left(k_{1}\right) \psi\left(k_{2}\right) \psi^{\dagger}\left(k_{3}\right) \psi^{\dagger}\left(k_{4}\right) .
\end{aligned}
$$

Our convention is that $\lambda<0$ represents an attractive interaction. Only two kinematic configurations are marginal in the

\footnotetext{
${ }^{5}$ It is interesting to note that, similarly to (2.12), in the soft collinear effective theory (SCET) of QCD there are also additional logarithms from collinear divergences.
}

presence of a Fermi surface: forward scattering (FS), where the angle between the incoming pair of fermions is the same as that for the outgoing pair, and BCS, where the initial angles are opposite [4]. In $d=2$, the forward-scattering constraint has the simple form $\theta_{1}=\theta_{3}$ and $\theta_{2}=\theta_{4}$, or its permutation. Then the coupling becomes a function $\lambda\left(\theta_{1}-\theta_{2}\right)$ of the relative angle between the incoming fermions. Instead, $\theta_{1}=-\theta_{2}$ and $\theta_{3}=-\theta_{4}$ for BCS, with the coupling now being a function $\lambda\left(\theta_{1}-\theta_{3}\right)$.

In the spherical RG, the angle on the Fermi surface plays the role of a flavor index for the low-energy excitations. It is then convenient to write this coupling function in a basis of spherical harmonics,

$$
\lambda_{L}=\int_{0}^{2 \pi} \frac{d \theta}{\sqrt{2 \pi}} e^{i L \theta} \lambda(\theta),
$$

which will have simple properties under renormalization. (Here $\theta=\theta_{12}$ or $\theta_{13}$ for forward scattering and BCS, respectively). The generalization to $d=3$ uses Legendre polynomials, and $\cos \theta_{i j}=\vec{k}_{i} \cdot \vec{k}_{j} / k_{F}^{2}$. In a local analysis on the Fermi surface, one can equivalently choose a basis of plane waves. Such a local analysis will typically be appropriate for our renormalization of the forward-scattering four-fermion interactions. We will see in a moment that this is because the exchange of the massless boson at momenta below the cutoff $\Lambda_{b}$ corresponds to scattering between nearby (i.e., nearly collinear) directions on the Fermi surface, with a change in angles of order $\Lambda_{b} / k_{F}$. For this reason, and also for simplicity, in what follows, we will use such a plane wave basis.

Consider now the effect of integrating out a shell of highmomentum bosons; this generates an effective $\psi^{4}$ interaction

$$
\begin{aligned}
S_{4-\text { Fermi, eff }}= & -\frac{g^{2}}{2} \int_{q, p, p^{\prime}} \frac{\delta K\left(q^{2} / \Lambda_{b}^{2}\right)}{q_{0}^{2}+\vec{q}^{2}} \\
& \times \psi^{\dagger}(p+q) \psi(p) \psi^{\dagger}\left(p^{\prime}-q\right) \psi\left(p^{\prime}\right),
\end{aligned}
$$

where the variation $\delta K$ of the smooth cutoff function has the effect of restricting $q$ to a shell of size $\delta \Lambda_{b}$. In the notation of (2.13), this generates a 4-Fermi coupling

$$
\lambda\left(p^{\prime}-q, p+q, p^{\prime}, p\right)=-\frac{g^{2}}{2} \frac{\delta K\left(q^{2} / \Lambda_{b}^{2}\right)}{q_{0}^{2}+\vec{q}^{2}},
$$

and the FS and BCS marginal channels correspond to

$$
\mathrm{FS}: p \approx p^{\prime}-q, \quad \mathrm{BCS}: p \approx-p^{\prime} .
$$

Notice that the fermion momenta $p$ and $p^{\prime}$ are of order $k_{F}$, while the exchanged boson momentum $|\vec{q}| \sim \Lambda_{b} \ll k_{F}$. This effective 4-Fermi interaction then couples patches of the Fermi surface over small angular sizes $\sim \Lambda_{b} / k_{F}$ (nearly tangential patches for FS, and antipodal for BCS). As we anticipated above, this is the reason why we can restrict to a local analysis on the Fermi surface over angular scales $\sim \Lambda_{b} / k_{F}$.

The question we need to address is how to absorb this momentum-dependent vertex into a renormalization of constant couplings. This is accomplished by changing to the angular momentum basis, defined, in a local patch near the Fermi surface, as

$$
\lambda_{\vec{L}}=\int \frac{d^{2} q_{\|}}{(\sqrt{2 \pi})^{2}} e^{i \vec{L} \cdot \vec{q}_{\|} / k_{F}} \lambda(q) .
$$



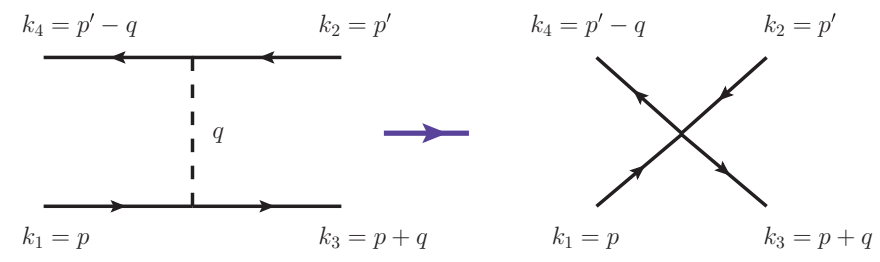

FIG. 3. (Color online) Boson-mediated $\psi^{4}$ interaction at tree level. The FS channel is $p \approx p^{\prime}-q$, and the BCS channel is $p \approx-p^{\prime}$.

This generalizes (2.14) to $d=3$, and $q$ is the difference in the momenta of incoming fermions. (On the full spherical Fermi surface, the plane waves are replaced by Legendre polynomials, as discussed before.) In this basis, the momentumdependent vertex (2.16) amounts to a change

$$
\begin{aligned}
\delta \lambda_{L} & =g^{2} \int \frac{d^{2} q_{\|}}{(\sqrt{2 \pi})^{2}} \frac{e^{i \vec{L} \cdot \vec{q}_{\|} / k_{F}}}{q_{0}^{2}+q_{\perp}^{2}+q_{\|}^{2}} \delta K\left(q^{2} / \Lambda_{b}^{2}\right) \\
& \approx g^{2} J_{0}\left(|L| \Lambda_{b} / k_{F}\right) \frac{\delta \Lambda_{b}}{\Lambda_{b}} .
\end{aligned}
$$

The Bessel function $J_{0}$ decays and oscillates for large $|L|$ and thus logarithmic running will effectively be turned off exponentially quickly for $\Lambda_{b}|L| \gg k_{F}$.

This is depicted in Fig. 3. It is important to stress that in the effective 4-Fermi interaction (2.15), the exchanged momentum $q$ is restricted to a shell of high momenta. This in turn constrains the angular integration in the spherical harmonic decomposition to $\Delta \theta=\Lambda_{b} / k_{F}<1$, which is the origin of the $\ln \Lambda_{b}$ dependence that we just encountered.

Therefore, already at tree level we need to add a counterterm for $\left(\psi^{\dagger} \psi\right)^{2}$ in order to cancel this divergence and have a finite physical coupling. We write the bare coupling in terms of the physical coupling plus a counterterm:

$$
\lambda_{0, L}=\lambda_{L}+\delta \lambda_{L},
$$

and choose

$$
\delta \lambda_{L}=g^{2} \ln \Lambda_{b}
$$

for $|L|<k_{F} / \Lambda_{b}$. Higher modes do not run. Equivalently, one can work in dimensional regularization (DR). Since this is a mass-independent scheme, there is no upper bound on the $|L|$ for which $\lambda_{L}$ gets a logarithmic divergence and thus $\delta \lambda_{L}=$ $g^{2} / \epsilon$ for all $\vec{L}$. It requires taking $k_{F} \rightarrow \infty$ from the beginning, before taking $\epsilon \rightarrow 0$, and thus formally $|L| / k_{F}$ is always below the $\mathrm{RG}$ scale $\mu$; this is fine as long as one is restricted to $|L|<k_{F} / \mu$. We conclude that there is a tree-level running of the $\psi^{4}$ coupling,

$$
\beta_{\lambda_{L}}=-\frac{d \lambda_{L}}{d \ln \Lambda_{b}}=g^{2} .
$$

An attractive coupling ( $\lambda<0$ in our convention) then grows towards the IR. Our analysis has been for general fermion momenta $p$ and $p^{\prime}$, so the tree-level $\beta$ function (2.22) applies to both FS and BCS channels of 4-Fermi interactions.

The existence of a tree-level $\beta$ function for $\lambda_{L}$ is special to finite density. This does not occur in zero-density relativistic theories, although effects such as that of Fig. 3 are formally generated by a Wilsonian exact RG [13]. Even in the exact Wilsonian RG, in the relativistic case one can always eliminate such tree-level running terms by an extra step where their effects are reproduced by loop-level terms. In our context, the tree-level logarithmic enhancement is a consequence of exchanging high-momentum bosons that induce virtual scatterings tangential to the Fermi surface, so that modes far below the cutoff can exchange a mode near the cutoff. Since the diagram in the figure is not one-particle irreducible, this is an example of a situation where the 1PI action and the Wilsonian action are different. In the following sections such tree-level contributions will be shown to be key for understanding the $\mathrm{RG}$ of the quantum theory.

\section{RENORMALIZATION OF THE QUANTUM THEORY AT ONE LOOP}

In this section we prove the renormalizability of the theory at one loop, and calculate the RG $\beta$ functions. This requires evaluating the one-loop corrections to the boson and fermion self-energies, as well as the renormalization of the $\phi \psi^{\dagger} \psi$ and $\left(\psi^{\dagger} \psi\right)^{2}$ vertices. The vacuum polarization of the boson is finite and has been calculated in detail in many places (see, e.g., [18-20]), and will not be repeated here. We will return to its effects in higher loop diagrams in Sec. V.

We will find that the one-loop RG is scheme-dependent, both on the regularization (cutoff versus DR) and on the renormalization subtraction. Scheme dependence in finitedensity QFT starts at one loop due to the presence of tree-level running - in the relativistic context, this would usually be seen only from two loops (and in theories with a single coupling, only from three loops). For computational purposes, it is always more convenient to work in DR, and this is indeed the scheme where we originally found many of the effects that will be described shortly. However, it turns out that the standard minimal subtraction scheme in DR leads to unphysical results for the RG (such as wrong sign anomalous dimensions), because it misses important running effects in the quadratic action from finite pieces. For this reason, in this section we will present the results mostly with the cutoff regulator of Sec. II B and in a physical subtraction scheme. Dimensional regularization of the finite-density theory will be studied in the Appendix. We will also check that both regulators agree on a physical subtraction, as it should be.

\section{A. Ward identities near the Fermi surface}

The field theory has a conserved charge associated with complex rotations of the fermions, which becomes a gauge symmetry if a dynamic electromagnetic field is added. The expression for the current in the low-energy theory is

$$
J_{\mu}(p)=\psi^{\dagger}(p) V_{\mu} \psi(p)+O\left(p_{\perp} / k_{F}\right) .
$$

We have introduced the velocity 4-vector

$$
V_{\mu} \equiv(i,-v \hat{n})
$$

such that $V \cdot p=i p_{0}-v \hat{n} \cdot \vec{p}$ is the fermion kinetic term in the effective theory. Varying the direction $\hat{n}=\vec{p} /|\vec{p}|$ obtains an infinite number of approximately conserved currents, each associated with the effective theory on a small Fermi surface 

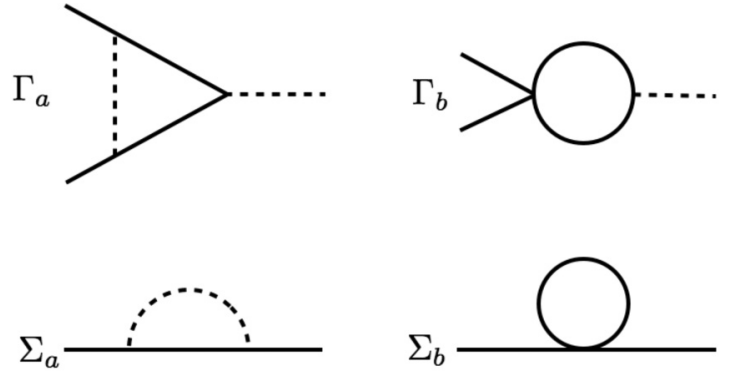

FIG. 4. One-loop diagrams that renormalize the cubic vertex and fermion self-energy.

patch (this neglects curvature corrections and interpatch couplings).

Quantum-mechanically, the conserved current leads to a Ward identity relating the 3-point function $\left\langle J_{\mu} \psi^{\dagger} \psi\right\rangle$ to the fermion self-energy; see, e.g., [21]. In more detail, defining

$$
\left\langle J_{\mu}(q) \psi^{\dagger}(p+q) \psi(p)\right\rangle=\Gamma_{\mu}(p ; q) G(p) G(p+q),
$$

the Ward identity reads

$$
q_{\mu} \Gamma_{\mu}(p ; q)=G^{-1}(p)-G^{-1}(p+q) .
$$

Here $G$ is the full fermion propagator, related to the tree-level expression and the self-energy by

$$
G^{-1}=G_{0}^{-1}-\Sigma \text {. }
$$

In a relativistic theory, taking the limit $q \rightarrow 0$ leads to the infinitesimal version of the Ward identity

$$
\Gamma_{\mu}=\partial_{\mu} \Sigma .
$$

However, we will see that at finite density the limits $q_{0} \rightarrow 0$ and $|\vec{q}| \rightarrow 0$ do not commute; this makes the infinitesimal identity ambiguous, but (3.4) is still well defined. Similar ambiguities are found in QCD at finite density [22,23].

Equation (3.4) can be used to relate the quantum corrections to the Yukawa vertex ${ }^{6}$

$$
\Gamma(p ; q)=-\left\langle\phi(q) \psi^{\dagger}(p+q) \psi(p)\right\rangle-g
$$

and the fermion self-energy. In the approximation (3.1), the Yukawa vertex is related to the current vertex (3.3) by

$$
\Gamma_{\mu} \approx V_{\mu} \Gamma / g
$$

and plugging this into (3.4) then obtains

$$
\Gamma(p ; q)=g \frac{\Sigma(p+q)-\Sigma(p)}{V \cdot q}+O\left(q_{\perp} / k_{F}\right),
$$

a result that will be important for our renormalization approach.

\section{B. Vertex renormalization and fermion self-energy}

Let us now calculate the one-loop corrections to the fermion self-energy and Yukawa vertex, shown in Fig. 4. This analysis

\footnotetext{
${ }^{6}$ Our convention is that $(g+\Gamma) \phi \psi^{\dagger} \psi$ gives the renormalized vertex.
}

will clarify the origin of singular operators generated at the quantum level. In our calculations we use renormalized perturbation theory [see (2.7)], and adjust the counterterms in order to cancel loop divergences.

\section{Vertex correction}

The one-loop vertex correction $\Gamma_{a}$ of Fig. 4 is, linearizing the fermion propagators around the Fermi surface,

$$
\begin{aligned}
\Gamma_{a}(k ; q) \approx & \frac{g^{3}}{(2 \pi)^{4}} \int \frac{d p_{0} d p_{\perp} d^{2} p_{\|}}{\left(k_{0}-p_{0}\right)^{2}+\left(k_{\perp}-p_{\perp}\right)^{2}+p_{\|}^{2}} \\
& \times \frac{1}{i p_{0}-v p_{\perp}} \frac{1}{i\left(p_{0}+q_{0}\right)-v\left(p_{\perp}+q_{\perp}\right)} .
\end{aligned}
$$

The momenta have been decomposed with respect to the external fermion momentum $\vec{k}=\hat{n}\left(k_{F}+k_{\perp}\right)$ as follows: $\vec{p}=$ $\hat{n}\left(k_{F}+p_{\perp}\right)+p_{\|}$and $\vec{q}=\hat{n} q_{\perp}+q_{\|}$.

There are two types of contributions to the vertex correction. First there is a regular piece $\Gamma_{\text {reg }}$ that comes from evaluating (3.10) setting $q=0$ inside the integral. This is dominated by the region of large momenta around $\Lambda_{f}$ in Fig. 2. However, there is an additional divergence, that is not accounted for in the usual treatment [3]. This singular piece $\Gamma_{\text {sing }}=\Gamma-\Gamma_{\text {reg }}$ comes from a small region $p_{\perp}+q_{\perp} \approx 0$ around the origin where the two fermion poles are on opposite sides of the real axis. This is the contribution from the $\delta$ function peak on the Fermi surface from the product of two fermion lines, that we discussed before in (2.9). Normally such terms would not contribute to the RG, but in our model they are logarithmically enhanced due to the exchange of virtual bosons, and should be included. ${ }^{7}$

The regular and singular contributions to the vertex depend on the regularization scheme, and here we evaluate these using the cutoff prescription set up in Sec. II B. The DR expressions are given in the Appendix. In

$$
\Gamma_{\text {reg }}=\frac{g^{3}}{(2 \pi)^{4}} \int \frac{d p_{0} d p_{\perp} d^{2} p_{\|}}{p_{0}^{2}+p_{\perp}^{2}+p_{\|}^{2}} \frac{1}{\left(i p_{0}-v p_{\perp}\right)^{2}}
$$

we have to integrate over $p_{0}$ first; integrating then over $p_{\|}$(this is convergent and can be extended to $\Lambda_{b} \rightarrow \infty$ ) and finally over $p_{\perp}$ with cutoff $\Lambda_{f}$ yields

$$
\Gamma_{\text {reg }}=\Gamma\left(\hat{n} k_{F} ; 0\right) \approx \frac{g^{3}}{4 \pi^{2}} \frac{\ln \Lambda_{f}}{1+|v|},
$$

up to finite terms. The singular contribution comes from the $\delta$-function peak on the Fermi surface from the product of two fermion lines, that we discussed before in (2.9). It evaluates to

$$
\Gamma_{\text {sing }}\left(\hat{n} k_{F} ; q\right) \approx \frac{g^{3}}{4 \pi^{2}} \frac{\operatorname{sgn}(v) q_{\perp}}{i q_{0}-v q_{\perp}} \ln \Lambda_{b} .
$$

Equation (3.13) is quite puzzling because it contains a logarithmic divergence for a singular ${ }^{8}$ operator. At face value,

\footnotetext{
${ }^{7}$ Another model where singular contributions play a role in the renormalization of the theory is [24].

${ }^{8}$ By singular (sometimes also referred to as "nonlocal") we mean that the operator has a singular dependence on frequency/momenta.
} 
this would imply that the theory is not renormalizable. One could argue that the nonlocal term is not Wilsonian-it comes from the $\delta$-function peak in (2.9) right on the Fermi surfaceand hence should not be included in the RG. However, ignoring logarithms is dangerous: in the IR such effects grow and the Wilsonian prediction will not be a good approximation to the physical answer. Moreover, in higher loop diagrams nonlocal divergences will mix with local ones and, again, these effects cannot be ignored. Physically, we have a UV-IR mixing, where an IR contribution from excitations at the Fermi surface multiplies a UV enhancement from high-momentum modes.

However, we will now show that the solution lies in the second diagram $\Gamma_{b}$ of Fig. 4, which will precisely cancel the nonlocal divergence. This diagram has not been included in previous RG approaches, since the fermion loop from the forward-scattering 4-Fermi interaction is finite-in fact, it vanishes if the fermion is restricted to a high-momentum shell as in [4]. In the present case, however, the situation is different because already at tree level the 4-Fermi interaction has a logarithmic divergence. Inserting this into the one-loop diagram, and taking into account the $\delta$-function peak in (2.9), will give the right contribution to make the renormalization procedure well defined. The reason behind this cancellation is that the region of fermion momenta that gives rise to the nonlocal renormalization is the same one where boson exchange leads to the effective 4-Fermi vertex through the process of Fig. 3.

Let us see how this comes about. Denoting the momenta in the external fermion lines by $p$ and $k$, so that there is an incoming boson momentum $k-p$, diagram $\Gamma_{b}$ in Fig. 4 gives

$$
\begin{aligned}
\Gamma_{b}(p, k ; k-p)= & -g \int d^{2} L\left(\lambda_{L}+\delta \lambda_{L}\right) \int \frac{d^{d+1} q}{(2 \pi)^{d+1}} \\
& \times e^{i \overrightarrow{q_{\|}} \cdot \vec{L} / k_{F}} G(k+q) G(p+q), \quad(3
\end{aligned}
$$

where the overall minus sign comes from the fermion loop. The 4-Fermi vertex is in the FS channel, but we will avoid writing explicitly the "FS" subscript in this section, as this is the only type of vertex that will appear. ${ }^{9}$

The $d^{2} \vec{q}_{\|}$integration is done trivially since the fermion propagators depend only on $q_{\perp}$, and only the zero mode $\lambda_{L=0}$ contributes. The remaining integration is finite but ambiguous, which also reflects the scheme dependence that we found before. In the regularization with $\Lambda_{b}$ and $\Lambda_{f}$, the integral over frequencies is done first as in (2.9), obtaining

$$
\begin{aligned}
\Gamma_{b}(p, k ; k-p)= & -\left(\lambda_{L=0}+\delta \lambda_{L=0}\right) g \frac{\operatorname{sgn}(v)}{4 \pi^{2}} \\
& \times \frac{k_{\perp}-p_{\perp}}{i\left(k_{0}-p_{0}\right)-v\left(k_{\perp}-p_{\perp}\right)} .
\end{aligned}
$$

Combining this with $\Gamma_{a}$ in (A3), and using $\delta \lambda_{L=0}=g^{2} \ln \Lambda_{b}$ for the counterterm [see (2.21)], the nonlocal divergences

\footnotetext{
${ }^{9}$ In the applications in Sec. IV the BCS vertex will also enter, and then we will distinguish this channel explicitly.
}

precisely cancel and we arrive at

$$
\Gamma(p ; q) \approx \frac{g^{3}}{4 \pi^{2}} \frac{1}{1+|v|} \ln \Lambda_{f}-\frac{g \lambda_{L=0}}{4 \pi^{2}} \frac{\operatorname{sgn}(v) q_{\perp}}{i q_{0}-v q_{\perp}} .
$$

Note that the divergence in the singular contribution (3.13) is a $\ln \Lambda_{b}$. This illustrates the more general point that the seemingly nonlocal renormalization effects found in [3] are controlled by $\Lambda_{b}$. The running velocity below will be another example of this.

\section{Fermion self-energy}

The one-loop fermion self-energy turns out to be the most delicate renormalized quantity. First, with a cutoff regulator the result for $\Sigma_{a}$ is very sensitive to the way in which the fermion loop momentum is regulated. This problem is fortunately avoided in DR, and we present the corresponding calculation in the Appendix. Additionally, by power-counting there is a linear divergence in $\Sigma_{b}$ that complicates the regularization of the subleading logarithmically divergent kinetic terms. This affects both the cutoff and dimensional regulators.

The diagram $\Sigma_{a}$ is evaluated in detail in the Appendix, where we also show explicitly that it satisfies the Ward identity that relates it to $\Gamma_{a}$. We will then not repeat the analog calculation with cutoffs, and simply deduce $\Sigma_{a}$ from (3.12) plus (3.13) using (3.9):

$$
\Sigma_{a}(q)=\frac{g^{3}}{4 \pi^{2}} \frac{\ln \Lambda_{f}}{1+|v|}\left(i q_{0}-v q_{\perp}\right)+\frac{g^{3}}{4 \pi^{2}} \ln \Lambda_{b} \operatorname{sgn}(v) q_{\perp} .
$$

Next, the diagram $\Sigma_{b}(k)$ in Fig. 4 is proportional to $\lambda_{L=0} \int d q_{0} d q_{\perp} G(k+q)$. This integral is ambiguous in the low-energy theory where the linear dispersion relation is used, and we need to regularize it in a way consistent with the Ward identity. As alluded to above, the computation of $\Sigma_{b}(k)$ is complicated by the linear divergence. As this may be completely absorbed by a counterterm, we can without loss of generality compute the less-divergent quantity $\Sigma_{b}(k)-\Sigma_{b}(0)$ :

$$
\begin{aligned}
& \Sigma_{b}(k)-\Sigma_{b}(0) \propto \lambda_{L=0} \int d q_{0} \int d q_{\perp} \\
& \quad \times\left(\frac{1}{i\left(q_{0}+k_{0}\right)-v\left(q_{\perp}+k_{\perp}\right)}-\frac{1}{i q_{0}-v q_{\perp}}\right) .
\end{aligned}
$$

In the cutoff approach we are instructed to perform the convergent $q_{0}$ integration first and obtain

$$
\int d q_{\perp}\left[\Theta\left(q_{\perp}+k_{\perp}\right)-\Theta\left(q_{\perp}\right)\right]=k_{\perp} .
$$

However, it is easy to see by inspection that the $d q_{\perp}$ integrand in (3.18) vanishes if we shift the $q_{\perp}$ integration variable of $\Sigma_{b}(k)$ by $-k_{\perp}$ relative to $\Sigma_{b}(0)$. In fact, (3.18) has an ambiguity that is exactly parametrized by this relative shift $a_{\perp}$ in the 
integration variable $q_{\perp}$ :

$$
\begin{aligned}
\Sigma_{b}(k)-\Sigma_{b}(0) & \propto \lambda_{L=0} \int d q_{0} \int d q_{\perp}\left(\frac{1}{i\left[q_{0}+k_{0}+a_{0}(k)\right]-v\left[q_{\perp}+k_{\perp}+a_{\perp}(k)\right]}-\frac{1}{i q_{0}-v q_{\perp}}\right) \\
& \propto \lambda_{L=0} \int d q_{\perp}\left[\Theta\left(q_{\perp}+k_{\perp}+a_{\perp}(k)\right)-\Theta\left(q_{\perp}\right)\right]=\lambda_{L=0}\left[a_{\perp}(k)+k_{\perp}\right] .
\end{aligned}
$$

An identical ambiguity arises in the evaluation of triangle diagrams for anomalies in relativistic theories. Here, as there, the parameter $a_{\perp}(k)$ represents an additional piece of data that must be input into the theory, either from matching to a UV theory or by constraints on the low-energy theory. Taylorexpanding $a_{\perp}(k)$ in $k$, we can discard the constant piece since $\Sigma_{b}(0)-\Sigma_{b}(0)=0$, and furthermore on dimensional grounds we should discard terms of $O\left(k_{0}^{2}, k_{\perp}^{2}\right)$ and higher as well. We are left with $a_{\perp}(k)=a_{1} k_{0}+a_{2} k_{\perp}$ and hence

$$
\Sigma_{b}(k)=-\frac{\lambda_{L=0}+\delta \lambda_{L=0}}{4 \pi^{2}} \operatorname{sgn}(v)\left[\left(a_{1} k_{0}+a_{2} k_{\perp}\right)+k_{\perp}\right] .
$$

For a given regulator, there is a unique choice for $a_{1}, a_{2}$ that respects gauge invariance. In the present case, we see that the Ward identity (3.9) holds only for $a_{\perp}(k)=0$. Thus,

$$
\begin{gathered}
\Sigma_{b}(k)=-\frac{\lambda_{L=0}+\delta \lambda_{L=0}}{4 \pi^{2}} \operatorname{sgn}(v) k_{\perp}, \\
\Sigma(k) \approx \frac{g^{2}}{4 \pi^{2}} \frac{\ln \Lambda_{f}}{1+|v|}\left(i k_{0}-v k_{\perp}\right)-\frac{\lambda_{L=0}}{4 \pi^{2}} \operatorname{sgn}(v) k_{\perp} .
\end{gathered}
$$

The first term in $\Sigma(k)$ is a wave function renormalization; notice, however, that the logarithmic divergences for velocity renormalization have canceled between $\Sigma_{a}$ and $\Sigma_{b}$, with the result that the velocity renormalization is finite and proportional to $g \lambda_{L=0}$. This is analogous to the cancellation of the nonlocal terms in the vertex. Therefore, unlike [3,25], we find no UV divergence for the velocity after taking into account the tree-level running of the 4-Fermi vertex.

This however highlights an important point about the running of terms in the quadratic action, specifically the velocity and the wave-function renormalization. Below, we will discuss how a physical subtraction scheme for the fermion self-energy does produce a running velocity. More generally, it guarantees that the full physical logarithmic enhancement of $q_{0}$ and $q_{\perp}$ terms in $\Sigma$ gets taken into account in the running velocity $v$ and wave-function factor $Z$, and therefore get fully included in the fermion propagator. This is a significant advantage of physical subtraction; the alternative would require calculations of physical amplitudes to include a large number of diagrams with insertions of the $\Sigma_{b}$ diagram from Fig. 4 as subdiagrams, which would quickly become very difficult. For the wave-function factor, this is even more important, because its running produces anomalous dimensions that feed into the running of all parameters. Thus, in practice one should always define the wave-function renormalization counterterm $\delta Z$ so that it agrees with physical subtraction, and we believe it is vastly easier and more transparent to define the running of $v$ this way as well.

\section{Comments on singular operators}

Having calculated the one-loop contributions to the fermion self-energy and cubic vertex, it will be useful now to clarify more the origin of the singular contributions that we have found.

Focusing on the self-energy (3.23), the first term corresponds to wave-function renormalization $Z_{\psi}$, while the second term amounts to a correction to the velocity. We can combine both into a single momentum-dependent renormalization factor $\mathcal{Z}(k)$, rewriting the quantum effect as

$$
L_{\text {fermion, kin }}=-\mathcal{Z}(k) \psi^{\dagger}\left(i k_{0}-v k_{\perp}\right) \psi
$$

with

$$
\mathcal{Z}(k)=\frac{g^{2}}{4 \pi^{2}} \frac{\ln \Lambda_{f}}{1+|v|}-\frac{\lambda_{L=0}}{4 \pi^{2}} \frac{\operatorname{sgn}(v) k_{\perp}}{i k_{0}-v k_{\perp}} .
$$

Therefore, from the point of view of the original fermion dispersion relation, the velocity renormalization corresponds to a singular contribution to $\mathcal{Z}(k)$. We stress that the running of $v$ is a direct consequence of the UV-IR mixing discussed above: the IR enhancement of excitations near the Fermi surface multiplying a UV contribution from the exchange of virtual bosons tangential to the Fermi surface. Without these effects, the RG would be analytic and the velocity would not run.

By the Ward identity, the factor $\mathcal{Z}(k)$ is the same as the vertex correction $\Gamma$, the correlation function that originally displayed the nonlocal contributions. The key point is that these effects are controlled by the physical 4-Fermi coupling $\lambda_{L}$ and do not require introducing a new coupling or counterterm, something that would have obstructed the renormalization of the theory. Moreover, in terms of the two RG scales introduced in Sec. II B, their running is set by $\ln \mu_{b}$, the boson scale.

The poles in (3.25) or (3.16) contain information about the physical spectrum of excitations near the Fermi surface. Similar singularities arise in the 4-Fermi vertex in the Fermi liquid, and should be treated in the same way [17]. The difference here is the logarithmic enhancement from boson exchange, reflected in the running of the physical coupling $\lambda_{L}$. Let us illustrate this with the set of one-loop contributions to fermion scattering shown in Fig. 5.

The diagram from boson exchange gives a $\ln ^{2} \Lambda_{b}$, which is canceled by the insertion of the counterterm $\delta \lambda_{L}$ in the other
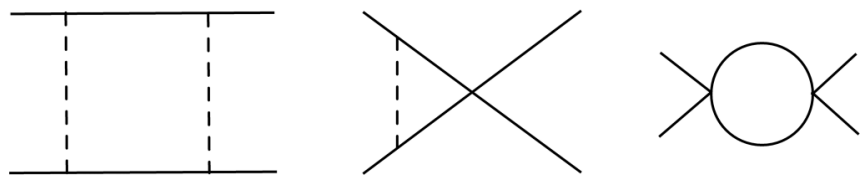

FIG. 5. One-loop contributions to the zero-sound (forwardscattering) vertex. 
two diagrams, with the result that the sum of the three diagrams depends only on the physical coupling $\lambda_{L}$. Denoting the two fermion momenta for the forward-scattering channel by $p$ and $k$ obtains

$$
\begin{aligned}
\Gamma^{(4)}(p, k) & =-\lambda^{2} \int \frac{d^{d+1} q}{(2 \pi)^{d+1}} G(k+q) G(p+q) \\
& =-\frac{\lambda^{2}}{4 \pi^{2}} \frac{\operatorname{sgn}(v)\left(p_{\perp}-k_{\perp}\right)}{i\left(p_{0}-k_{0}\right)-v\left(p_{\perp}-k_{\perp}\right)} .
\end{aligned}
$$

This gives a singular contribution to the zero-sound (forwardscattering) vertex, which is familiar from quantum treatments of Fermi liquids [17].

Our point again is that this singular contribution is still controlled by the local coupling $\lambda\left(\mu_{b}\right)$ and does not induce an independent RG flow. Had we not included the tree-level running of $\lambda$, the cancellation of logarithmic divergences would have failed, and we would have found a $1 n^{2}$ multiplying a singular momentum-dependent function. The physical content here is the same as that for the renormalization of the velocity and the singular contribution to the cubic vertex, all of them being related by the Ward identity. It is not consistent to ignore these singular contributions, by the same reason that we cannot ignore the renormalization of the velocity.

\section{RG $\beta$ functions}

We are now ready to put these results together and determine the RG flow of the theory at one loop. ${ }^{10}$ We stress again that in finite-density QFT one generically expects scheme dependence already at one loop, due to the tree-level running of the 4-Fermi coupling. We have seen this before in the differences between the dimensional and cutoff regulators, and below additional scheme dependence will arise from the renormalization conditions.

Let us adopt a physical renormalization scheme where the renormalized couplings are defined in terms of physical amplitudes at an RG scale $\mu$. For simplicity we also scale the two cutoffs in the same way and denote them by $\Lambda$; we briefly discuss the bidimensional RG below. Defining $t=\ln \mu / \Lambda$, from (3.23) we read off the counterterms

$$
\delta_{\psi}=\frac{g^{2}}{4 \pi^{2}(1+|v|)} t, \quad \delta v=-\frac{\operatorname{sgn}(v) \lambda_{L=0}}{4 \pi^{2}}
$$

and recalling that $\beta_{\lambda_{L}}=g^{2}$ obtains the anomalous dimension and running velocity

$$
\gamma_{\psi}=\frac{g^{2}}{8 \pi^{2}(1+|v|)}, \quad \beta_{v}=\frac{\operatorname{sgn}(v) g^{2}}{4 \pi^{2}} .
$$

On the other hand, for the vertex correction (3.16) we may define $g$ as the amplitude $\Gamma(p, q)$ evaluated at the point

$$
q_{0}=x \mu, \quad q_{\perp}=\mu .
$$

\footnotetext{
${ }^{10}$ It will be useful to recall our sign conventions for the quantum corrections and counterterms. The corrections to the fermion kinetic term appear in the combination $\delta_{\psi}\left(i p_{0}-v p_{\perp}\right)-\delta v p_{\perp}+\Sigma$, while for the cubic vertex we have $g+\delta g+\Gamma$.
}

Then, we identify

$$
\delta g=\frac{g^{3}}{4 \pi^{2}(1+|v|)} t-\frac{g \lambda_{L=0}}{4 \pi^{2}} \frac{\operatorname{sgn}(v)}{v} \frac{1}{1-i x / v} .
$$

In calculating $\beta_{g}$, the first term in $\delta g$ cancels against the anomalous dimension; however, the second term in $\delta g$ now gives a nonzero contribution proportional to $\beta_{\lambda_{L=0}}$. The $\beta$ function for the theory in $d=3-\epsilon$ becomes

$$
\begin{aligned}
\beta_{g}(x) & =-\frac{\epsilon}{2} g+\frac{g}{4 \pi^{2}} \frac{\operatorname{sgn}(v)}{v} \frac{1}{1-i x / v} \beta_{\lambda_{L=0}} \\
& =-\frac{\epsilon}{2} g+\frac{g^{3}}{4 \pi^{2}|v|} \frac{1}{1-i x / v} .
\end{aligned}
$$

We are free to choose any value for the ratio $x$ at the physical subtraction point; for instance the choice $x=\infty$ removes the last term.

The $x$-dependent $\beta$ function (3.31) may look puzzling at first, but we should stress that this is fairly generic of physical subtraction schemes. In fact, it can arise even in relativistic $\lambda \phi^{4}$ theory, where choosing a physical subtraction scheme of say $\mathcal{M}_{2 \rightarrow 2}\left(s_{0}, t_{0}, u_{0}\right)=\lambda$ for the $2 \rightarrow 2$ amplitude $\mathcal{M}_{2 \rightarrow 2}$ at some subtraction point $s_{0}, t_{0}, u_{0}$ would lead to a dependence of the $\beta$ function for $\lambda$ on the ratio of Mandelstam variables $s_{0} / t_{0}, s_{0} / u_{0}$ at sufficiently high loop order. This just reflects that in a physical subtraction scheme, one by definition subtracts off from the bare couplings $\lambda_{B}=\lambda+\delta \lambda$ any difference between the amplitude $\mathcal{M}\left(s_{0}, t_{0}, u_{0}\right)$ and the renormalized coupling $\lambda$ itself. Since the finite part of the amplitude depends on $s, t$ times the running couplings, in order to do such a subtraction one is forced to subtract off a nontrivial running function of the subtraction point. In other words, this encodes the fact that the finite part of the amplitude contains nontrivial dependence on dimensionless kinematic ratios (here, $s / t$ or $q_{0} / q$ ) times running couplings. Another example where momentum-dependent effects play an important role in the RG is in bicritical systems, where the $\beta$ functions depend explicitly on the ratio between momenta and the symmetry-breaking mass [26]. ${ }^{11}$

It is generally stated in the literature that for the Abelian theory in $d=3$, the $\beta$ function $\beta_{g}$ vanishes identically due to the Ward identity. However, in this case this statement should be interpreted with some care. Given the renormalization of the velocity, the Ward identity implies a $\beta$ function (3.31) that depends on the ratio $x=q_{0} / q_{\perp}$ at the subtraction point. In the regime where $x \rightarrow \infty$, the $\beta$ function does vanish, but more generally $\beta_{g}(x) \neq 0$. So one must be precise about what limits of momenta one uses to define the running coupling if one wishes to obtain certain features of the $\beta$ function.

We may also interpret these results using the bidimensional $\mathrm{RG}$ of Sec. II B. In this case, the nonsingular RG evolution is controlled by $\mu_{f}$, while singular effects evolve along $\mu_{b}$ :

$$
\frac{\partial v}{\partial t_{b}}=\frac{\operatorname{sgn}(v) g^{2}}{4 \pi^{2}}, \quad \frac{\partial g}{\partial t_{b}}=\frac{g^{3}}{4 \pi^{2}} \frac{\operatorname{sgn}(v)}{i x-v} .
$$

We expect that at higher loop order the RG flows along $t_{b}$ and $t_{f}$ will start to mix. Furthermore, in generalizations of our

\footnotetext{
${ }^{11} \mathrm{We}$ thank the anonymous referee for pointing this out to us.
} 

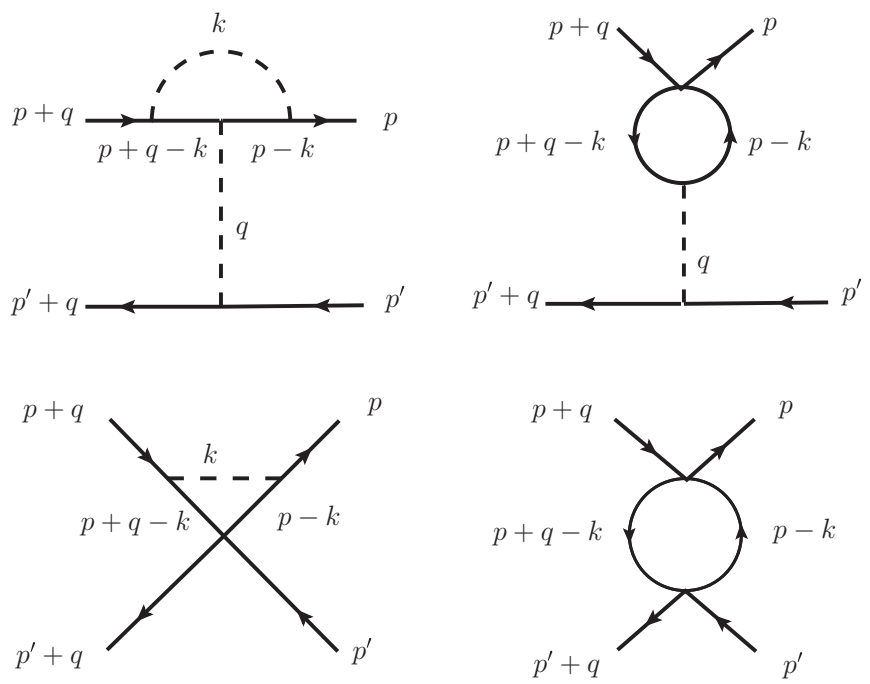

FIG. 6. Diagrams contributing to the renormalization of the forward-scattering Landau parameter at one loop.

theory with matrix-valued $\phi$, the anomalous dimension and $\delta g$ contributions will not cancel, with the result that $g$ evolves along both RG directions simultaneously. Of course, we can always project this bidimensional RG flow down to $t_{b} \propto t_{f}$, but having the two different scales helps to track the physical origin of the different quantum contributions.

\section{APPLICATIONS}

So far we have analyzed the renormalization of the Fermi surface coupled to a gapless boson, focusing on the anomalous dimension, running velocity, and vertex corrections. These operators can be defined and studied in a local patch, and are approximately insensitive to global properties of the Fermi surface. ${ }^{12}$ There are, however, other observables of interest, where the quasiparticles can exchange large momenta of order $k_{F}$. In this section we will briefly consider the renormalization of these quantities, applying the approach described in Secs. II D and III.

One of the most important operators of this type is the 4Fermi BCS interaction. In the presence of the massless scalar, this leads to a parametric enhancement in the condensation of Cooper pairs [6,7]. The RG approach to Fermi surface instabilities will be discussed in detail in [9]. Here we will instead consider the renormalization of Landau parameters in Sec. IV A and the $2 k_{F}$ vertex in Sec. IV B, which are closely related to our analysis in the previous sections.

\section{A. Renormalization of Landau parameters}

As the first application of the Wilsonian RG that we have proposed, we will analyze in more detail the renormalization of the 4-Fermi FS coupling. The diagrams that contribute at one loop are shown in Fig. 6. Note that the zero-sound diagrams of Fig. 5 vanish for the forward-scattering coupling on the Fermi

\footnotetext{
${ }^{12}$ Recall that the effective theory keeps bosons of momenta $|p|<$ $\Lambda_{b}$, so the angular size of one of these patches is set by $\Lambda_{b} / k_{F}$.
}

surface, as the poles from the fermion propagators are always on the same side of the complex plane.

The first diagram in the figure has a one-loop vertex correction inserted as a subdivergence in the tree-level running of $\lambda$. This will give a logarithmic divergence with both regular and singular terms, as in (3.12) and (3.13). In the theory with a singlet scalar $\phi$ that we have discussed so far, the regular term cancels exactly against the anomalous dimension contribution. However, the anomalous dimension dominates for a theory with matrix-valued $\phi$, where $\psi$ and $\phi$ transform in the fundamental and adjoint representations, respectively. Next, the singular divergence will cancel against the second diagram on top, as in (3.16). Finally, combining with the two diagrams in the bottom of the figure will cancel the $\ln \Lambda_{b}$ divergence from the integral over the boson propagator, replacing it by the physical coupling $\lambda_{L}$. In summary, in the singlet-scalar $\phi$ theory

$$
\beta_{\lambda_{L}}(x)=g^{2}+\frac{g^{2}}{2 \pi^{2}|v|} \frac{1}{1-i x / v} \lambda_{L}
$$

with $x=q_{0} / q_{\perp}$. The first term is the tree-level running from boson exchange, and the net contribution from the anomalous dimension appears in the matrix-valued $\phi$ case. The origin of the last term is the same as in (3.31).

This result of our RG approach is important both conceptually and for its possible consequences. If the interaction is defined right at the Fermi surface, $x \rightarrow \infty$ and the one-loop correction vanishes. However, in various processes it is natural to consider a static interaction at finite momentum, in which case $x=0$ and we find a net one-loop renormalization of the Landau parameter. This same contribution will be obtained in the adiabatic limit $v / c \gg 1$ at fixed $x$. Similar effects can be seen in the BCS channel.

The $\beta$ function at $x=0$ also appears to have interesting applications. Indeed, for an attractive interaction we could balance the tree-level relevant contribution against the oneloop irrelevant term, finding an approximate fixed point at $\left|\lambda_{L}\right|=2 \pi^{2}|v|$. Of course, this simple analysis will be subject to various corrections (such as those from Landau damping and superconductivity), and it is not our goal here to model-build a controlled fixed point. But the possibility of approximate fixed points for the Landau parameters would lead to interesting novel phases, and we hope to return to this point in future work.

\section{B. Friedel oscillations and the $2 k_{F}$ vertex}

A weakly coupled Fermi liquid has singularities in the density-density correlator $\langle\rho(k) \rho(-k)\rangle$ at $k=2 k_{F}$. The reason is that a scattering process with large momentum transfer $\vec{K}=$ $2 k_{F} \hat{n}$ can take a quasiparticle at the Fermi surface with $\vec{p}=$ $-k_{F} \hat{n}$ into another one with $\vec{p}+\vec{K}=k_{F} \hat{n}$, which is also at the Fermi surface. These $2 k_{F}$ singularities produce $\sin \left(2 k_{F} r\right)$-type oscillations in position space, known as Friedel oscillations. The density-density correlator is then an important gaugeinvariant probe of the structure of the Fermi surface. This observable also appears in systems with electric impurities interacting with conduction electrons, where it controls the linear response to the presence of impurities.

An important goal is to understand the structure of $2 k_{F}$ singularities in strongly interacting systems. Previous 

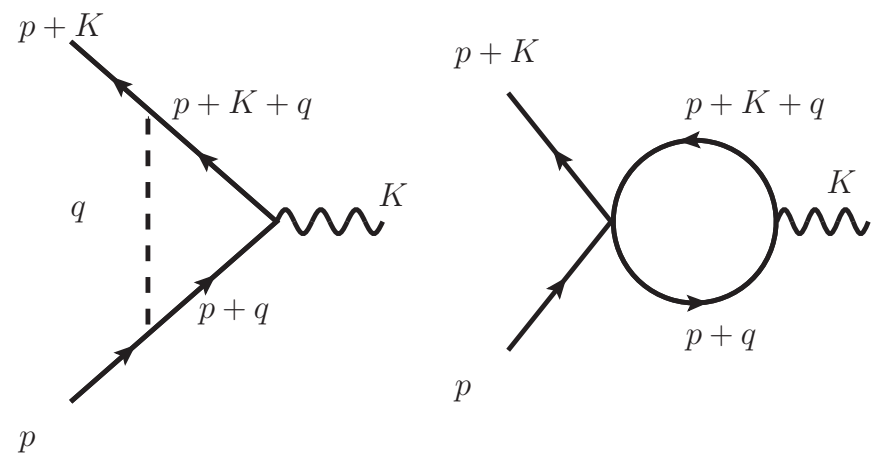

FIG. 7. One-loop renormalization of the $2 k_{F}$ vertex, represented by the insertion of the wavy line with momentum $|K|=2 k_{F}$.

calculations in $2+1$ dimensions include [11,27,28]. A puzzling aspect of the renormalization of the $2 k_{F}$ response is that double logarithms appear already at one loop. Here we will study the renormalization of the $2 k_{F}$ vertex using our $\mathrm{RG}$ approach, and we will see how this problem is resolved. Very similar computations appear in [9] for the BCS and CDW instabilities.

Let us consider the $2 k_{F}$ vertex in our perturbative theory, which arises from coupling the fermion density to an external gauge field of momentum $K=2 k_{F},{ }^{13}$

$$
L_{2 k_{F}}=-i \int \frac{d^{d} p}{(2 \pi)^{d}} u_{K} \psi^{\dagger}(p+K) \psi(p) .
$$

As before, the renormalization is carried out by distinguishing between the bare and renormalized coupling,

$$
u_{K, 0}=\mu^{1+\epsilon / 2} \frac{Z_{u}}{Z_{\psi}} u_{K},
$$

where the power of the RG scale is the classical dimension in $d=3-\epsilon$ and $Z_{\psi}$ accounts for the wave function renormalization of the fermions. It is convenient to define $Z_{u} u_{K}=u_{K}+\delta u_{K}$, and the vertex including quantum effects will be denoted by

$$
\mu^{1+\epsilon / 2}\left(u_{K}+\delta u_{K}+\Gamma_{K}\right)=-i\left\langle\psi^{\dagger}(\vec{K} / 2) \psi(-\vec{K} / 2)\right\rangle .
$$

The one-loop contributions to $\Gamma_{K}$ are shown in Fig. 7 .

We analyze first the diagram with the virtual boson and $\vec{p}=-\vec{K} / 2$, given by

$$
\begin{aligned}
\Gamma_{K}^{(1)}= & g^{2} u \int \frac{d^{d+1} q}{(2 \pi)^{d+1}} D(q) \frac{1}{i q_{0}-\varepsilon(-K / 2+q)} \\
& \times \frac{1}{i q_{0}-\varepsilon(K / 2+q)} .
\end{aligned}
$$

This has the same structure as the vertex correction of Sec. III B, except that the large boson momentum transfer reverses the direction of the Fermi velocity. As a result, the quantum correction will be quite different. Given $\vec{K}=2 k_{F} \hat{n}$, we decompose $\vec{q}=q_{\perp} \hat{n}+q_{\|}$. Assuming for simplicity a

\footnotetext{
${ }^{13}$ The overall factor of $-i$ is the Euclidean convention for the $A_{0}$ component.
}

quadratic dispersion relation obtains

$$
\begin{aligned}
\Gamma_{K}^{(1)}= & \frac{g^{2} u}{(2 \pi)^{3}} \int \frac{d q_{0} d q_{\perp} q_{\|} d q_{\|}}{q_{0}^{2}+q_{\perp}^{2}+q_{\|}^{2}} \frac{1}{i q_{0}+v q_{\perp}-\frac{v}{2 k_{F}} \vec{q}^{2}} \\
& \times \frac{1}{i q_{0}-v q_{\perp}-\frac{v}{2 k_{F}} \vec{q}^{2}} .
\end{aligned}
$$

We will analyze the regularization and renormalization of $u_{K}$, and also take the opportunity to spell out in more detail several general aspects of our renormalization prescription. For one, like most of the diagrams in this paper, once the $\frac{v}{2 k_{F}} \vec{q}^{2}$ terms are included in the fermion propagator, $\Gamma_{K}^{(1)}$ is UV-convergent as can be seen by power counting. However, in this case UV-convergence is a drawback since there are large logarithms in the IR that one would like to resum using the RG. ${ }^{14}$ This is accomplished by working in the low-momentum effective theory where the $\frac{v}{2 k_{F}} \vec{q}^{2}$ terms are Taylor expanded and treated in the quadratic fermion action as interaction terms rather than terms that are included in the propagator:

$$
\begin{aligned}
\frac{1}{i q_{0}+v q_{\perp}-\frac{v}{2 k_{F}} \vec{q}^{2}} \rightarrow & \frac{1}{i q_{0}+v q_{\perp}}+\frac{1}{i q_{0}+v q_{\perp}} \\
& \times \frac{v}{2 k_{F}} \vec{q}^{2} \frac{1}{i q_{0}+v q_{\perp}}+\cdots .
\end{aligned}
$$

For the cutoff regulator which is more physically transparent, this just requires taking $\Lambda_{b} \ll \sqrt{2 k_{F} \mu_{f}}$ with $\mu_{f}$ the RG scale $\mu_{f}^{2} \sim q_{0}^{2}+v^{2} q_{\perp}^{2}$. At large $k_{F}$ and fixed $\Lambda_{b}$, this condition is clearly satisfied. The higher derivative terms in (4.7) are then negligible, and the integrals are regulated by our cutoff prescription instead and not by the quadratic part of the dispersion relation. For the dimensional regulator, we formally take the limit $k_{F} \rightarrow \infty$ inside the fermion propagator first, and then $\epsilon \rightarrow 0$.

The integral (4.6) has an infrared divergence that is regulated by taking finite external momenta. In practice, since we are not specifically interested here in the dependence on this momenta and just want to see the renormalization procedure working, it will be simpler to regulate the infrared by adding small mass terms. Furthermore, although it is conceptually useful to use a separate cutoff $\Lambda_{b}$ for bosons and $\Lambda_{f}$ for fermions, for the example in this section we find it simpler to use a single cutoff $\Lambda>\sqrt{q_{0}^{2}+q_{\perp}^{2}+q_{\|}^{2}}$ for both. ${ }^{15}$ We will therefore evaluate the log-enhanced parts of the following integral:

$$
\begin{aligned}
\Gamma_{K}^{(1)}= & -\frac{g^{2} u}{(2 \pi)^{3}} \int_{q_{0}^{2}+q_{\perp}^{2}+q_{\|}^{2}<\Lambda^{2}} \\
& \times \frac{d q_{0} d q_{\perp} q_{\|} d q_{\|}}{\left(q_{0}^{2}+q_{\perp}^{2}+q_{\|}^{2}+\mu^{2}\right)\left(q_{0}^{2}+v^{2} q_{\perp}^{2}+\mu^{2}\right)} \\
= & -\frac{g^{2} u}{(2 \pi)^{2} v}\left[\frac{1}{2} \ln ^{2} \Lambda-\ln \left(\frac{1+v}{2 v} \mu\right) \ln \Lambda+O\left(\Lambda^{0}\right)\right] .
\end{aligned}
$$

\footnotetext{
${ }^{14}$ We thank Catherine Pepin for emphasizing to us this obstacle in some previous treatments.

${ }^{15}$ It is easy to see that the tree-level logarithmic divergence of $\lambda$ is the same with this cutoff as with $\Lambda_{b}$.
} 
One way to evaluate this integral is by converting $d q_{0} d q_{\perp}$ to radial coordinates:

$$
\begin{aligned}
\Gamma_{K}^{(1)} & =-2 \frac{g^{2} u}{(2 \pi)^{3}} \int_{0}^{1} d x x^{-1 / 2} y^{-1 / 2} \int_{0}^{\Lambda} \rho d \rho \int_{0}^{\sqrt{\Lambda^{2}-\rho^{2}}} q d q \frac{1}{\left[\mu^{2}+\rho^{2}\left(x+v^{2} y\right)\right]\left(\rho^{2}+q^{2}+\mu^{2}\right)} \\
& =-\frac{g^{2} u}{(2 \pi)^{3}} \int_{0}^{1} d x \frac{-\operatorname{Li}_{2}\left(-\frac{\left(y v^{2}+x\right)\left(\Lambda^{2}+\mu^{2}\right)}{\left(1-v^{2}\right) y \mu^{2}}\right)+\operatorname{Li}_{2}\left(1-\frac{1}{\left(1-v^{2}\right) y}\right)+\ln \left(\frac{\mu^{2}}{\Lambda^{2}+\mu^{2}}\right) \ln \left(\frac{1}{\left(1-v^{2}\right) y}\right)}{2 \sqrt{x y}\left(v^{2} y+x\right)} \\
& \stackrel{\Lambda \rightarrow \infty}{\rightarrow}-\int_{0}^{1} d x\left[\ln ^{2} \Lambda\left(\frac{1}{4 \sqrt{x y}\left(v^{2} y+x\right)}\right)+\ln \Lambda\left(\frac{\ln \left(\frac{v^{2} y+x}{\mu^{2}}\right)}{\sqrt{x y}\left(v^{2} y+x\right)}\right)+O\left(\Lambda^{0}\right)\right],
\end{aligned}
$$

where $y \equiv 1-x$. Performing the $d x$ integration gives (4.8). $\Gamma_{K}^{(2)}$ can be evaluated in a similar way to $\Gamma_{K}^{(1)}$ :

$$
\begin{aligned}
\Gamma_{K}^{(2)} & =2 \frac{u}{(2 \pi)^{3}}\left[\lambda+g^{2} \ln \left(\frac{\Lambda}{M}\right)\right] \int_{0}^{1} d x x^{-1 / 2} y^{-1 / 2} \int_{0}^{\Lambda} \rho d \rho \frac{1}{\left[\mu^{2}+\rho^{2}\left(x+v^{2} y\right)\right]} \\
& =\frac{u}{(2 \pi)^{3}}\left[\lambda+g^{2} \ln \left(\frac{\Lambda}{M}\right)\right] \int_{0}^{1} d x x^{-1 / 2} y^{-1 / 2} \frac{1}{x+v^{2} y} \ln \frac{\left(x+v^{2} y\right) \Lambda^{2}+\mu^{2}}{\mu^{2}},
\end{aligned}
$$

and the final integration produces (4.11).

There is also a contribution from the BCS channel (since $p$ and $p+K$ are nearly opposite) four-Fermi interaction $\lambda_{L=0}^{\mathrm{BCS}}$ and its tree-level counterterm $\delta \lambda_{L=0}^{\mathrm{BCS}}=g^{2} \ln \left(\frac{\Lambda}{M}\right)$, where $M$ is the RG scale:

$$
\begin{aligned}
\Gamma_{K}^{(2)} & =\frac{u}{(2 \pi)^{3}}\left[\lambda_{L=0}^{\mathrm{BCS}}+g^{2} \ln \left(\frac{\Lambda}{M}\right)\right] \int_{q_{0}^{2}+q_{\perp}^{2}<\Lambda^{2}} \frac{d q_{0} d q_{\perp}}{q_{0}^{2}+v^{2} q_{\perp}^{2}+\mu^{2}} \\
& =\frac{u}{(2 \pi)^{2} v}\left[g^{2} \ln \frac{\Lambda}{M} \ln \Lambda+\lambda_{L=0}^{\mathrm{BCS}} \ln \Lambda-g^{2} \ln \left(\frac{1+v}{2 v} \mu\right) \ln \frac{\Lambda}{M}+O\left(\Lambda^{0}\right)\right] .
\end{aligned}
$$

We see explicitly that the $\ln \mu \ln \Lambda$ term cancels in the sum $\Gamma_{K}^{(1)}+\Gamma_{K}^{(2)}$ (which is crucial since $\mu$ is playing the role analogous to that of external momenta):

$$
\begin{aligned}
\Gamma_{K}^{(1)}+\Gamma_{K}^{(2)} & =\frac{u}{(2 \pi)^{2} v}\left[\frac{1}{2} g^{2} \ln ^{2} \Lambda+\left[\lambda_{L=0}^{\mathrm{BCS}}-g^{2} \ln (M)\right] \ln \Lambda+O\left(\Lambda^{0}\right)\right] \\
& =\frac{u}{(2 \pi)^{2} v}\left[\frac{1}{2} g^{2} \ln ^{2} \frac{\Lambda}{M}+\lambda_{L=0}^{\mathrm{BCS}} \ln \frac{\Lambda}{M}+O\left(\Lambda^{0}\right)\right] .
\end{aligned}
$$

From this, we read off that the counterterm for $u$ at this order must be

$$
\delta u=-\frac{u}{(2 \pi)^{2} v}\left(\frac{1}{2} g^{2} \ln ^{2} \frac{\Lambda}{M}+\lambda_{L=0}^{\mathrm{BCS}} \ln \frac{\Lambda}{M}\right)+\text { finite }
$$

where the finite $\Lambda$-independent piece is scheme-dependent and we will choose it to vanish.

The $\beta$ function for the dimensionless coupling $u$ can be determined by the condition that the bare term $u_{0}=M(u+$ $\delta u-u \delta Z_{\psi}$ ) is independent of the RG scale $M$ :

$$
\begin{aligned}
0 & =M \frac{d}{d M} u_{0}=M \frac{d}{d M}\left[M\left(u+\delta u-u \delta Z_{\psi}\right)\right] \\
& =u+\beta_{u}-2 \gamma_{\psi} u+M \frac{d}{d M} \delta u,
\end{aligned}
$$

where $\beta_{u}=M \frac{d}{d M} u$. Collecting terms in an expansion in powers of $\ln \frac{\Lambda}{M}$, we therefore have

$$
\begin{aligned}
0= & \left(u+\beta_{u}-2 \gamma_{\psi} u+\frac{u \lambda_{L=0}^{\mathrm{BCS}}}{(2 \pi)^{2} v}\right) \\
& +\ln \frac{\Lambda}{M} \frac{u}{(2 \pi)^{2} v}\left(-g^{2}+\beta_{\lambda}+\cdots\right)+\cdots,
\end{aligned}
$$

where “..." denotes higher orders in $\ln \frac{\Lambda}{M}$ and/or couplings. We can read off the $\beta_{u}$ function from the cancellation of the $\Lambda$-independent piece,

$$
\beta_{u}=u\left(-1+2 \gamma_{\psi}-\frac{\lambda_{L=0}^{\mathrm{BCS}}}{(2 \pi)^{2} v}\right),
$$

and the cancellation in the coefficients of the higher powers of $\ln \frac{\Lambda}{M}$ provides consistency conditions that will be satisfied due to the running from lower-order diagrams. In particular, we see from the linear in $\ln \left(\frac{\Lambda}{M}\right)$ term in (4.15) that $\beta_{\lambda}=g^{2}$. So we see here that even if we had not thought to consider tree-level running of $\lambda$, we would have noticed it must be included just from analysis of the diagrams in Fig. 7.

Equation (4.16) is our final result for the renormalization of the $2 k_{F}$ vertex, showing how multilogarithms are properly taken into account by the running of the couplings. The fermion anomalous dimension (3.28) always makes the vertex irrelevant, and the same is true for an attractive $\lambda_{L=0}<0$ in- 
(a)

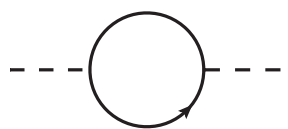

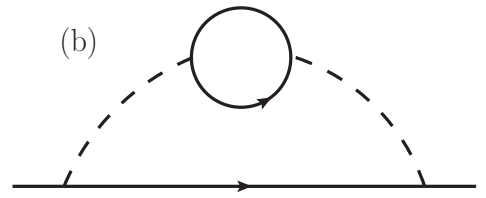

FIG. 8. (a) Landau damping for the boson self-energy. (b) Twoloop correction to the fermion self-energy with a boson self-energy insertion.

teraction (generated, e.g., by the exchange of high-momentum bosons). We conclude that in systems with gapless spin-zero bosons, perturbative quantum corrections tend to smooth out the $2 k_{F}$ singularities. In contrast, the sign of the 4-Fermi contribution is reversed in a theory with gauge fields, ${ }^{16}$ so there can be a competition between the vertex correction and $\gamma_{\psi}$. On a different note, holographic models at finite density have signatures of Fermi surfaces that are strongly suppressed $[29,30]$, and it is interesting that our perturbative results also point in the same direction. It would be important to try to continue this calculation to strong coupling, and to apply these results to models with impurities.

\section{RENORMALIZATION AT HIGHER ORDERS AND LANDAU DAMPING}

So far we have established the renormalizability of the theory of a Fermi surface coupled to a massless boson at one-loop order, and have set up a consistent (in the sense of including the dominant quantum effects) Wilsonian RG framework. We expect that our approach for organizing divergences and the $\mathrm{RG}$ can also be extended to higher loop level without obstruction, though at present we have no general proof. A new element to take into account is that when going to higher orders it is necessary to include Landau damping. We will now explain how to extend our RG to include such effects. ${ }^{17}$

Landau damping effects come from the one-loop fermion bubble that contributes to the boson self-energy, Fig. 8(a). This diagram is finite, and that is why formally it did not affect the one-loop RG; crucially, however, the large Fermi momentum $k_{F}$ reappears here when integrating over the Fermi surface, with the result that (a) becomes important at a high scale proportional to $g k_{F}$. This in turn gives two-loop corrections to the RG, such as diagram (b) in Fig. 8, that are comparable or can even dominate over the one-loop results. At this point, the standard perturbative RG breaks down. The failure of the perturbative expansion is due to the fact that the loop factor $g^{2} / 16 \pi^{2}$ from the additional boson self-energy insertion in (b) also comes with a power of $k_{F}^{2}$ from the integral over the Fermi surface.

\footnotetext{
${ }^{16}$ The gauge field, being a vector, couples with opposite signs to fermions with opposite Fermi velocity. This is unlike the scalar field, which couples with the same sign to all patches. This was also observed in [28].

${ }^{17}$ Since a detailed analysis of Landau damping and how it affects fermion correlation functions was recently performed in [3], our discussion here will be brief.
}

A well-known solution to this problem is to reorganize the perturbative expansion in terms of the resummed boson propagator,

$$
\begin{aligned}
D^{-1}(p) & =p_{0}^{2}+\vec{p}^{2}+\Pi(p), \\
\Pi(p) & =M_{D}^{2} \frac{p_{0}}{v|\vec{p}|} \tan ^{-1} \frac{v|\vec{p}|}{p_{0}},
\end{aligned}
$$

with

$$
M_{D}^{2}=\frac{g^{2} k_{F}^{2}}{2 \pi^{2} v},
$$

and $\Pi(p)$ is the result of evaluating diagram (a). Physically, Landau damping comes from virtual particle/hole pairs near the Fermi surface, whose contribution is given by integrating the $\delta$-function peak (2.9) over the Fermi surface (up to a constant term that needs to be fixed in order to tune the boson to criticality). We have seen before that for consistency the $\mathrm{RG}$ needs to include this region near the Fermi surface (see, e.g., Fig. 2), and it is satisfying that this same prescription also captures Landau damping. Furthermore, note that at low energies the propagator can be approximated by

$$
D^{-1}(p) \approx \vec{p}^{2}+\frac{\pi}{4} M_{D}^{2} \frac{p_{0}}{v|\vec{p}|}
$$

giving a boson with $z=3$ dynamical exponent $[18,19]$.

From the point of view of the original theory, this resummation amounts to including an infinite class of diagrams, and it is important to determine when this is consistent. Within our perturbative framework with small coupling $g$ and near three dimensions this appears to be the case. Examination of diagrams reveals that (a) in the figure gives the dominant nonanalytic contribution responsible for the $z=3$ scaling, and that other effects are perturbative analytic corrections on this. It would be interesting to understand this more generally, but here we will assume (5.1) and study its consequences.

Let us for simplicity restrict our discussion to scales much smaller than

$$
\mu_{L D} \approx\left[v^{-1} \tan ^{-1}(v)\right]^{1 / 2} M_{D},
$$

where (5.3) is a good approximation. The one-loop fermion self-energy and vertex corrections (diagrams $\Sigma_{a}$ and $\Gamma_{a}$ in Fig. 4) using the Landau-damped propagator give [3]

$$
\Sigma_{a}(q) \approx i \frac{g^{2}}{12 \pi^{2}|v|} q_{0} \ln \frac{\Lambda}{q_{0}},
$$

as well as a nonlocal divergence

$$
\Gamma_{a}(k ; q)=\frac{g^{3}}{12 \pi^{2}|v|} \frac{i q_{0}}{i q_{0}-v q_{\perp}} \ln \frac{\Lambda}{q_{0}} .
$$

The factor of 3 difference with what we obtained above in Sec. III is a consequence of the boson dynamical exponent. Here we have for simplicity considered a single cutoff $\Lambda$, with $\Lambda_{b}^{3} \propto \Lambda_{f} \sim \Lambda$. The tree-level running similarly becomes $\beta_{\lambda_{L}}=g^{2} / 3$.

The renormalization now proceeds as before, by including the one-loop contributions $\Sigma_{b}$ and $\Gamma_{b}$ from the 4-Fermi interaction in Fig. 4. In particular, the fermion self-energy 
becomes

$$
\begin{aligned}
& \Sigma(q) \approx \frac{g^{2}}{12 \pi^{2}|v|} \ln \Lambda\left(i q_{0}-v q_{\perp}\right)-\frac{\lambda_{L=0}}{4 \pi^{2}|v|} v q_{\perp}, \\
& \Gamma(q) \approx \frac{g^{3}}{12 \pi^{2}|v|} \ln \Lambda-\frac{g \lambda_{L=0}}{4 \pi^{2}|v|} \frac{v q_{\perp}}{i q_{0}-v q_{\perp}} .
\end{aligned}
$$

In the overdamped regime the couplings always appear in combinations $g^{2} /|v|$ and $\lambda_{L} /|v|$ (as can be seen by redefining the fields), so it will be convenient to define

$$
\alpha \equiv \frac{g^{2}}{12 \pi^{2}|v|}, \quad \tilde{\lambda}_{L} \equiv \frac{\lambda_{L}}{|v|} .
$$

Using the physical renormalization scheme of Sec. III obtains the following $\beta$ functions:

$$
\begin{aligned}
& \gamma_{\psi}=\frac{\alpha}{2}, \quad \beta_{v}=\alpha v, \\
& \beta_{\tilde{\lambda}_{L}}=4 \pi^{2} \alpha-\alpha \tilde{\lambda}_{L}+\frac{2 \alpha}{1-i x / v} \tilde{\lambda}_{L}, \\
& \beta_{\alpha}=-\epsilon \alpha-\alpha^{2}+\frac{2 \alpha^{2}}{1-i x / v} .
\end{aligned}
$$

The origin of the $x=q_{0} / q_{\perp}$ dependent terms is the same as in (3.31) and (4.1).

For the renormalization condition $x \rightarrow \infty$ these $\beta$ functions do not admit fixed points. However, if $x=0$ the one-loop $\beta$ functions for $\alpha$ and $\tilde{\lambda}_{L}$ have zeros at $\alpha=\epsilon, \tilde{\lambda}_{L}=-4 \pi^{2}$. The latter is not under perturbative control, and it would be interesting if one could find an exact solution, or models where a small $\tilde{\lambda}_{L}$ can be achieved.

Finally, we note that in a large- $N$ generalization where $\phi$ is an $N \times N$ matrix and $\psi$ is a vector, the $x$ dependence is suppressed, and the $\alpha \tilde{\lambda}_{L}$ and $\alpha^{2}$ terms change sign. This parameter range gives the possibility of flowing to a nonFermi-liquid fixed point before reaching the superconducting scale, something that was briefly studied in [3] and that we plan to analyze in more detail in the future.

\section{FUTURE DIRECTIONS}

In this work we have studied finite-density QFT and established its renormalizability at one loop. We focused on the important example of a Fermi surface coupled to a gapless boson mode, and established an RG procedure that consistently takes into account the dominant quantum corrections. The key features of this approach are the tree-level running of all 4-Fermi interactions, together with a cutoff prescription that includes the UV-IR mixing of bosons and fermions. We also discuss how to partially extend the approach to higher loop order, by adding Landau damping to the RG.

Our results provide a framework where quantum corrections at finite density can be systematically calculated and incorporated into the RG. It would clearly be important to prove the renormalizability at all orders, perhaps generalizing [13] to finite density. It would also be interesting to apply our methods to the theory introduced in [31], with dimensional regulators $\epsilon_{\perp}$ and $\epsilon_{\|}$in both perpendicular and parallel directions to the Fermi surface. We anticipate nontrivial renormalization effects at finite $\epsilon_{\perp}$. Finally, we have seen the possibility of fixed points for 4-Fermi interactions. Such fixed points could lead to novel IR phases, and in future work we plan to explore this direction in more detail.

\section{ACKNOWLEDGMENTS}

We thank S. Kachru, J. Kaplan, M. Mulligan, and S. Raghu for interesting discussions about related subjects. G.T. is supported by CONICET and PIP Grant No. 11220110100752. H.W. is supported by a Stanford Graduate Fellowship.

\section{APPENDIX: DIMENSIONAL REGULARIZATION AT FINITE DENSITY}

In this Appendix we will study finite-density QFT in dimensional regularization, with $d=3-\epsilon$. This has the effect of analytically continuing the number of tangential directions to the Fermi surface, $d_{\|}=2-\epsilon$. We note that this is different from the dimensional regulator of $[32,33]$, where the normal directions to the Fermi surface are fractional.

\section{Vertex and fermion self-energy corrections}

Let us begin with the one-loop vertex correction $\Gamma_{a}$ of Fig. 4 in DR,

$$
\begin{aligned}
\Gamma_{a}(k ; q) \approx & \mu^{\epsilon} \frac{g^{3}}{(2 \pi)^{d+1}} \int \frac{d p_{0} d p_{\perp} d^{d-1} p_{\|}}{\left(k_{0}-p_{0}\right)^{2}+\left(k_{\perp}-p_{\perp}\right)^{2}+p_{\|}^{2}} \\
& \times \frac{1}{i p_{0}-v p_{\perp}} \frac{1}{i\left(p_{0}+q_{0}\right)-v\left(p_{\perp}+q_{\perp}\right)} .
\end{aligned}
$$

The regular term becomes

$$
\Gamma_{\text {reg }}=-\frac{g^{3}}{8 \pi^{2} \epsilon} \frac{1-|v|}{|v|(1+|v|)},
$$

and we can also calculate directly the total contribution [3]

$$
\Gamma_{a}\left(\hat{n} k_{F} ; q\right)=\frac{g^{3}}{4 \pi^{2}} \frac{1}{1+|v|} \frac{i q_{0}+\operatorname{sgn}(v) q_{\perp}}{i q_{0}-v q_{\perp}} \frac{1}{\epsilon}+O\left(\epsilon^{0}\right) .
$$

From here, the singular term is given by the difference

$$
\Gamma_{\text {sing }}=\Gamma-\Gamma_{\text {reg }}=\frac{g^{3}}{8 \pi^{2}|v| \epsilon} \frac{i q_{0}+v q_{\perp}}{i q_{0}-v q_{\perp}} .
$$

Notice that we can use this result for $\Gamma_{\text {sing }}$ to define how to evaluate $\int_{p} G(p) G(p+q)$ in DR, which by itself is not regulated by $\epsilon$. We will need this expression in order to calculate $\Gamma_{b}$. As explained in the main body of the text, for small $q$ it is enough to consider an ansatz

$$
G(p) G(p+q) \approx G(p)^{2}+f(q) \delta\left(p_{0}\right) \delta\left(p_{\perp}\right) .
$$

By definition, $\Gamma_{\text {sing }}$ is the piece that comes from this $\delta$-function term. Integrating (A5) and requiring that $f(q)$ reproduces $\Gamma_{\text {sing }}$ gives

$$
G(p) G(p+q) \approx G(p)^{2}+\frac{\pi i}{|v|} \frac{i q_{0}+v q_{\perp}}{i q_{0}-v q_{\perp}} \delta\left(p_{0}\right) \delta\left(p_{\perp}\right) .
$$

This should be contrasted with the cutoff prescription (2.9). From this point of view, DR corresponds to averaging the 
results of integrating over $p_{0}$ first, and integrating over $p_{\perp}$ first, something that makes sense as these coordinates are not distinguished.

With (A6) we can now evaluate $\Gamma_{b}$ :

$$
\Gamma_{b}=-\frac{g}{8 \pi^{2}|v|}\left(\lambda_{L=0}+\delta \lambda_{L=0}\right) \frac{i q_{0}+v q_{\perp}}{i q_{0}-v q_{\perp}} .
$$

Therefore, in DR the cancellation of divergences between $\Gamma_{a}$ and $\Gamma_{b}$ works out to give

$$
\Gamma(p ; q) \approx-\frac{g^{3}}{8 \pi^{2} \epsilon} \frac{1-|v|}{|v|(1+|v|)}-\frac{g \lambda_{L=0}}{8 \pi^{2}|v|} \frac{i q_{0}+v q_{\perp}}{i q_{0}-v q_{\perp}} \text {. (A }
$$

The one-loop divergent contributions to $\Gamma$ are hence different in the cutoff approach and in dimensional regularization, something to be expected on general grounds. Indeed, due to the tree-level running, scheme dependence in finite-density QFT with multiple couplings will occur at one loop. In our case, the divergent piece in dimensional regularization is somewhat unphysical, due to the apparent divergence for $v \rightarrow 0$ (which is canceled by the finite piece). The full correlator does not diverge in this limit, something that is explicit also in the cutoff result (3.16). In a physical subtraction scheme the cutoff and dimensional regulators will give the same results, as we verify shortly.

Let us now discuss the corrections to the fermion selfenergy. The first contribution $\Sigma_{a}$ in Fig. 4 gives $[3,25]$

$$
\begin{aligned}
\Sigma_{a}\left(k_{0}, \vec{k}\right) \approx & -g^{2} \mu^{\epsilon} \int \frac{d p_{0} d p_{\perp} d^{d-1} p_{\|}}{(2 \pi)^{d+1}} \frac{1}{p_{0}^{2}+p_{\perp}^{2}+p_{\|}^{2}} \\
& \times \frac{1}{i\left(p_{0}+k_{0}\right)-v\left(p_{\perp}+k_{\perp}\right)} \\
= & \frac{g^{2}}{4 \pi^{2}(1+|v|)}\left[i k_{0}+\operatorname{sgn}(v) k_{\perp}\right] \frac{1}{\epsilon}+O\left(\epsilon^{0}\right),
\end{aligned}
$$

with momenta $\vec{k}=\hat{n}\left(k_{F}+k_{\perp}\right), \vec{p}=\hat{n} p_{\perp}+\vec{p}_{\|}$. We see that (A3) and (A9) satisfy the Ward identity (3.9). From this point of view, the nonlocal term in $\Gamma_{a}$ is equivalent to a renormalization of the velocity, namely a self-energy that does not depend only on $i k_{0}-v k_{\perp}$.

For $\Sigma_{b}$ we will introduce separate dimensional regularization parameters $\epsilon_{\|}$and $\epsilon_{\perp}$ for the dimension and codimension, respectively, of the Fermi surface. The dimension of space is then $d=3-\epsilon_{\perp}-\epsilon_{\|}$. When we take the $\epsilon=\epsilon_{\perp}+\epsilon_{\|} \rightarrow 0$ limit, we shall first take $\epsilon_{\perp} \rightarrow 0$ followed by $\epsilon_{\|} \rightarrow 0$. As discussed in the main text, this leaves a finite shift ambiguity of the form (3.21). The Ward identity then fixes $a_{\perp}$, yielding

$$
\Sigma_{b}(k)=-\frac{\lambda_{L=0}+\delta \lambda_{L=0}}{8 \pi^{2}|v|}\left(i k_{0}+v k_{\perp}\right) .
$$

The final DR result for the self-energy is

$$
\begin{aligned}
\Sigma(k) \approx & -\frac{1}{8 \pi^{2}|v|}\left(\frac{1-|v|}{1+|v|} \frac{g^{2}}{\epsilon}+\lambda_{L=0}\right)\left(i k_{0}-v k_{\perp}\right) \\
& -\frac{\lambda_{L=0}}{4 \pi^{2}} \operatorname{sgn}(v) k_{\perp} .
\end{aligned}
$$

As noted above, in this scheme the apparent divergence at small velocities is canceled between the $1 / \epsilon$ and finite piece.

\section{2. $\beta$ functions}

In DR, the simplest subtraction scheme is minimal subtraction (MS), where the counterterms are defined to cancel only the $\epsilon$ pole. However, this scheme leads to unphysical results-in particular, from (A11) it leads to an anomalous dimension with the wrong sign. This is an artifact, which originates in neglecting the contribution from the physical coupling.

This motivates adopting a physical subtraction scheme for $\mathrm{DR}$, as we did with the cutoff regulator. Now the counterterms become

$$
\delta_{\psi}=\frac{1}{8 \pi^{2}|v|}\left(\frac{1-|v|}{1+|v|} \frac{g^{2}}{\epsilon}+\lambda_{L=0}\right), \quad \delta v=-\frac{\operatorname{sgn}(v) \lambda_{L=0}}{4 \pi^{2}},
$$

and we reproduce the anomalous dimension and running velocity (3.28). Similarly, from (A8),

$$
\delta g=-\frac{g^{3}}{8 \pi^{2}} \frac{1-|v|}{|v|(1+|v|)} \ln \mu-\frac{g \lambda_{L=0}}{8 \pi^{2}|v|} \frac{1+i x / v}{1-i x / v},
$$

at a renormalization point $q_{0}=x \mu, q_{\perp}=\mu$. The $\beta$ function computed from here agrees with the cutoff result (3.31).
[1] R. Mahajan, D. Ramirez, S. Kachru, and S. Raghu, Phys. Rev. B 88, 115116 (2013).

[2] A. L. Fitzpatrick, S. Kachru, J. Kaplan, and S. Raghu, Phys. Rev. B 88, 125116 (2013).

[3] G. Torroba and H. Wang, Phys. Rev. B 90, 165144 (2014).

[4] R. Shankar, Rev. Mod. Phys. 66, 129 (1994).

[5] J. Polchinski, arXiv:hep-th/9210046.

[6] D. T. Son, Phys. Rev. D 59, 094019 (1999).

[7] E. Shuster and D. Son, Nucl. Phys. B 573, 434 (2000).

[8] M. A. Metlitski, D. F. Mross, S. Sachdev, and T. Senthil, Phys. Rev. B 91, 115111 (2015).

[9] A. L. Fitzpatrick et al., arXiv:1410.6814.

[10] J. Polchinski, Nucl. Phys. B 422, 617 (1994).

[11] C. Nayak and F. Wilczek, Nucl. Phys. B 430, 534 (1994).
[12] K. Wilson and J. B. Kogut, Phys. Rep. 12, 75 (1974).

[13] J. Polchinski, Nucl. Phys. B 231, 269 (1984).

[14] S. J. Yamamoto and Q. Si, Phys. Rev. B 81, 205106 (2010).

[15] I. Stewart (unpublished).

[16] J.-Y. Chiu, A. Jain, D. Neill, and I. Z. Rothstein, J. High Energy Phys. 05 (2012) 084.

[17] A. A. Abrikosov, L. P. Gorkov, I. E. Dzyaloshinski, and I. E. Dzialoshinskii, Methods of Quantum Field Theory in Statistical Physics (Courier Dover Publications, New York, 1975).

[18] J. A. Hertz, Phys. Rev. B 14, 1165 (1976).

[19] A. J. Millis, Phys. Rev. B 48, 7183 (1993).

[20] N. Nagaosa, Quantum Field theory in Condensed Matter Physics (Springer-Verlag, New York, 1999). 
[21] S. Weinberg, Quantum Theory of Fields, Vol. 1, Foundations (Cambridge University Press, Cambridge, 1995).

[22] W. E. Brown, J. T. Liu, and H.-c. Ren, Phys. Rev. D 62, 054013 (2000).

[23] T. Schäfer and K. Schwenzer, Phys. Rev. D 70, 054007 (2004).

[24] A. Abanov, A. V. Chubukov, and J. Schmalian, arXiv:condmat/0107421.

[25] A. L. Fitzpatrick, S. Kachru, J. Kaplan, and S. Raghu, Phys. Rev. B 89, 165114 (2014).

[26] D. J. Amit and Y. Y. Goldschmidt, Ann. Phys. 114, 356 (1978).
[27] B. L. Altshuler, L. B. Ioffe, and A. J. Millis, Phys. Rev. B 50, 14048 (1994).

[28] D. F. Mross, J. McGreevy, H. Liu, and T. Senthil, Phys. Rev. B 82, 045121 (2010).

[29] J. Polchinski and E. Silverstein, Classical Quantum Gravity 29, 194008 (2012).

[30] T. Faulkner and N. Iqbal, J. High Energy Phys. 07 (2013) 060.

[31] I. Mandal and S.-S. Lee, arXiv:1407.0033.

[32] T. Senthil and R. Shankar, Phys. Rev. Lett. 102, 046406 (2009).

[33] D. Dalidovich and S.-S. Lee, Phys. Rev. B 88, 245106 (2013). 